\title{
Two Interacting Particles in a Random Potential: Mapping onto One Parameter Localization Theories without Interaction
}

\author{
Klaus Frahm ${ }^{(1,2)}$, Axel Müller-Groeling ${ }^{(1,3)}$ and Jean-Louis Pichard ${ }^{(1)}$ \\ ${ }^{1}$ Service de Physique de l'État condensé, CEA-Saclay, 91191 Gif-sur-Yvette Cedex, France \\ ${ }^{2}$ Instituut-Lorentz, University of Leiden, P.O. Box 9506, 2300 RA Leiden, The Netherlands \\ ${ }^{3}$ Max-Planck-Institut für Kernphysik, Postfach 1039 80, D-69029 Heidelberg, Germany
}

\begin{abstract}
We consider two models for a pair of interacting particles in a random potential: (i) two particles with a Hubbard interaction in arbitrary dimensions and (ii) a strongly bound pair in one dimension. Establishing suitable correpondences we demonstrate that both cases can be described in terms familiar from theories of noninteracting particles. In particular, these two cases are shown to be controlled by a single scaling variable, namely the pair conductance $g_{2}$. For an attractive or repulsive Hubbard interaction and starting from a certain effective Hamiltonian we derive a supersymmetric nonlinear $\sigma$ model. Its action turns out to be closely related to the one found by Efetov for noninteracting electrons in disordered metals. This enables us to describe the diffusive motion of the particle pair on scales exceeding the one-particle localization length $L_{1}$ and to discuss the corresponding level statistics. For tightly bound pairs in one dimension, on the other hand, we follow early work by Dorokhov and exploit the analogy with the transfer matrix approach to quasi-1d conductors. Extending our study to $M$ particles we obtain a $M-$ particle localization length scaling like the $M$ th power of the one-particle localization length.
\end{abstract}

PACS. 05.45+b, 72.15Rn 


\section{INTRODUCTION}

The concept of one parameter scaling pioneered by Thouless [1] and generalized by Abrahams et al [2, 3] has been very fruitful in the understanding of the localization problem for noninteracting electrons in a random potential. The relevant scaling parameter is the size-dependent conductance $g(L)$ (in units of $2 e^{2} / h$ ) determined by the so-called $\beta$-function $\beta(g)$ [2]. This $\beta$-function which contains all the relevant information has in principle to be calculated from a microscopic theory. However, the general field theoretical formulation in terms of a non linear $\sigma$ model [4,5] shows the universal behavior of $\beta(g)$ and that it only depends on the symmetry class and the space dimension. In $2+\varepsilon$ dimensions the $\beta$-function is accessible by diagrammatic perturbation theory [5 77], and in quasi-one dimension even "exact" solutions are known, implying exponential localization [8,5]. Furthermore, exact expressions for the first two moments of the conductance have been derived [9, 10].

In one or quasi-one dimension there is, apart from the $\sigma$ model, another powerful theory 111 to describe the scaling behavior of the conductance. In this approach the distribution of transmission eigenvalues is determined by a Fokker-Planck equation, the Dorokhov-MelloPereyra-Kumar (DMPK) equation, describing how the transmission properties evolve with the length of the wire. In the unitary symmetry class the entire distribution of transmission eigenvalues [12] and the first two conductance moments [13] are known for arbitrary length scales. Moreover, it has become clear recently that the DMPK equation and the $\sigma$ model formulation of quasi-1d transport are mathematically equivalent [13 [15]. In both approaches, one-parameter scaling emerges in a natural way.

Not long ago, Shepelyansky [16] studied the localization problem of two interacting particles (TIP). He considered a one-dimensional disordered potential and a local (Hubbard-like) repulsive or attractive interaction $U$. Assuming some generic properties of the interaction induced coupling matrix elements between localized one-electron product states, Shepelyansky found that certain TIP-states are extended on a scale given by the two-particle localization length $L_{2} \propto L_{1}^{2}$, which can be much larger than the one-particle localization length $L_{1}$. Using similar assumptions, Imry [17] was able to confirm Shepelyansky's original result for $L_{2}$ by using the Thouless [1] block scaling picture. He introduced the notion of a two-particle conductance $g_{2}(L)$ and assumed that it obeys the same scaling behavior as the one-particle conductance. In subsequent work, the enhancement of the TIP localization length was confirmed by numerical studies for the transfer matrix [18], the TIP-Hamiltonian [19] and the TIP-Green's function [20].

In this paper we show that both the $\sigma$ model and the Fokker-Planck approach can be succesfully applied to the TIP-localization problem, thus relating the TIP-problem to an equivalent problem without interactions. This allows us to identify a certain pair conductance $g_{2}$ associated with TIP-diffusion and TIP-localization and to show that $g_{2}$ is governed by the same scaling function as the one-electron conductance. Furthermore, we relate $g_{2}$ to the statistical properties of the TIP energy levels and to the transmission eigenvalues of the corresponding scattering problem.

In section [I] we consider, generalizing Shepelyansky, the TIP-problem in $d$ dimensions with a Hubbard-like repulsive or attractive interaction $U$. We first motivate an effective random matrix model that incorporates precisely the $2 d$-dimensional lattice structure of the two-electron pair states. The only assumptions concern the statistical independence and 
the typical size of the interaction-induced coupling matrix elements between these states. The next step is to map the random matrix model onto a supersymmetric non linear $\sigma$ model. The corresponding action is after appropriate identifications formally identical with the action of Efetov's "standard" $\sigma$ model [5] for disordered conductors. This allows us to determine in arbitrary dimensions the Breit-Wigner form for the local density of states, the different frequency regimes for the diffusion of interaction-assisted pairs at scale $L>L_{1}$, and the two point correlation for the corresponding TIP-levels. We relate $g_{2}$ to a pair Thouless energy $E_{c}^{(2)}$ defined as the energy range for finding Wigner-Dyson level statistics. However, we also obtain a subtle modification with respect to the one-particle problem: the diffusion is logarithmically suppressed and the size of the interaction-assisted pairs increases logarithmically with time. These results are in agreement with recent findings of Borgonovi and Shepelyansky [21,22] based on numerical and qualitative arguments. A summary of our main results has already been published in a separate publication [23] and we present here a comprehensive derivation of the different steps.

In section III, we consider a different TIP problem, which was first studied by Dorokhov [24]: a tightly bound pair of particles in a strictly $1 d$ random potential. In analogy to quasi one-dimensional conductors, we treat this problem in terms of the DMPK equation. The scattering channels for the transfer matrix formulation are now provided by the internal quantum modes of the bound pair. The conductance $g_{2}$ is then related to the transmission eigenvalues corresponding to these scattering channels. To identify $g_{2}$ in this approach, we have to determine one microscopic length scale, namely the effective mean free path or equivalently the localization length as a function of the microscopic parameters. Again we find that the "conductance" $g_{2}$ for tightly bound pairs has the same scaling with system size as the conductance of non interacting electrons in quasi one-dimensional wires. Extending this approach to $M$ bound particles in one dimension, we obtain a delocalization effect which increases with $M$ as $L_{1}^{M}$ if the particles are forced to stay within a distance of order $L_{1}$ from each other. The delocalization of certain $M$ particle states by a local interaction will be discussed in a greater generality elsewhere [25].

\section{TWO PARTICLES WITH LOCAL ATTRACTIVE OR REPULSIVE INTERACTIONS IN D-DIMENSIONS}

We consider two interacting electrons on a lattice in $d$ dimensions with site diagonal disorder and nearest neighbor hopping elements. We assume that all one electron eigenstates are localized with a typical localization length $L_{1} \gg 1$ (all length scales will be measured in units of the lattice constant). This requires the disorder to be sufficiently weak in one or two dimensions, while the disorder strength should be slightly above the critical disorder in three dimensions. The localized one electron states $\varphi_{\rho}(r)$ are labeled by an index $\rho$ describing the "center" of the state. We consider the case of symmetric two-electron wave functions $\psi\left(r_{1}, r_{2}\right)=\psi\left(r_{2}, r_{1}\right)$ (e.g. both electrons have opposite spin) and express the Hamilton operator in the basis of symmetrized products of one-electron states $\mid \rho_{1} \rho_{2}>$, given by the wave function $\left[\varphi_{\rho_{1}}\left(r_{1}\right) \varphi_{\rho_{2}}\left(r_{2}\right)+\varphi_{\rho_{1}}\left(r_{2}\right) \varphi_{\rho_{2}}\left(r_{1}\right)\right] / \sqrt{2}$ :

$$
H=\sum_{\rho_{1}, \rho_{2}}\left(\varepsilon_{\rho_{1}}+\varepsilon_{\rho_{2}}\right)\left|\rho_{1} \rho_{2}><\rho_{1} \rho_{2}\right|+\sum_{\rho_{1}, \rho_{2}, \rho_{3}, \rho_{4}} Q_{\rho_{1} \rho_{2} \rho_{3} \rho_{4}}\left|\rho_{1} \rho_{2}><\rho_{3} \rho_{4}\right|
$$


where $Q_{\rho_{1} \rho_{2} \rho_{3} \rho_{4}}$ are the interaction matrix elements. The quantities $\varepsilon_{\rho}$ are the one-electron energies of the localized states $\varphi_{\rho}(r)$. For a local Hubbard interaction with interaction strength $U$

$$
H_{\text {int }}=U \sum_{r}|r r><r r|
$$

we have for instance

$$
Q_{\rho_{1} \rho_{2} \rho_{3} \rho_{4}}=<\rho_{1} \rho_{2}\left|H_{i n t}\right| \rho_{3} \rho_{4}>=2 U \sum_{r} \varphi_{\rho_{1}}(r) \varphi_{\rho_{2}}(r) \varphi_{\rho_{3}}(r) \varphi_{\rho_{4}}(r) \quad .
$$

These interaction matrix elements become exponentially small if two of the "positions" $\rho_{j}$ are far away on a scale much larger than the one-electron localization length. Shepelyansky divided [16] (in one dimension) the problem described by the Hamiltonian (2.1) into two blocks: a diagonal one corresponding to matrix elements $\left\langle\rho_{1} \rho_{2}|H| \rho_{3} \rho_{4}\right\rangle$, where at least two of the positions $\rho_{j}$ are separated by more than $L_{1}$, and a random band matrix with a superimposed strong diagonal (SBRM), where every $\rho_{j}$ is at less than $L_{1}$ from the three others. The ensuing mathematical random matrix model has been investigated in [26 28]. The two crucial simplifications for this picture are first to neglect all exponentially small interaction matrix elements $Q_{\rho_{1} \rho_{2} \rho_{3} \rho_{4}}$ ( off-diagonal terms of the first block, off-diagonal terms outside the band of the second block, coupling terms between the two blocks) and second to assume that the remaining coupling elements (2.3) are independent random variables with typically the same size. Using the ansatz

$$
\varphi_{\rho}(r) \sim \frac{1}{\left(L_{1}\right)^{d / 2}} e^{-|r-\rho| / L_{1}} a_{\rho}(r) \quad,
$$

and applying a central limit theorem argument in (2.3), one may estimate [16, 17] the coupling strength between well coupled product states of the SBRM-block to be

$$
\left\langle Q_{\rho_{1} \rho_{2} \rho_{3} \rho_{4}}^{2}\right\rangle \sim U^{2} L_{1}^{-3 d} .
$$

In (2.4), we assume for simplicity that $a_{\rho}(r)$ is a random variable with $\left\langle a_{\rho}(r)\right\rangle=0$ and $\left\langle a_{\rho}(r) a_{\tilde{\rho}}(\tilde{r})\right\rangle=\delta_{\rho \tilde{\rho}} \delta_{r \tilde{r}}$.

\section{A. Effective Random Matrix Hamiltonian}

In this study, we will consider an effective random matrix Hamiltonian (ERMH), which relies only on the second of the above-mentioned simplifications. In other words, all product states $\mid \rho_{1} \rho_{2}>$ will be taken into account, and we keep the total Hamiltonian, and not only the SBRM sub-block where the states are strongly affected by the interaction. However, the coupling matrix elements (2.3) of the total Hamiltonian matrix will still be assumed to be independent (Gaussian) variables. This latter point is indeed quite a serious simplification since the correlations between different coupling matrix elements will be neglected. In particular, the exact operator identity $H_{i n t}^{2}=U H_{\text {int }}[$ see (2.2)] is violated. However, we believe that our ERMH will nevertheless be quite realistic due to the strongly fluctuating diagonal elements, which tend to eliminate the effect of the correlations. 
We consider a $2 d$-dimensional configuration space (lattice) with two coordinate vectors $R \equiv \rho_{1}+\rho_{2}$ and $j \equiv\left(\rho_{1}-\rho_{2}\right) / 2$ corresponding to twice the center of mass and half the distance of the two electrons. This choice ensures that the components of these coordinates differ for adjacent lattice sites just by +1 or -1 if the corresponding other coordinate is kept constant. The ERMH consists of two parts

$$
\mathcal{H}=\hat{\eta}+\hat{\zeta}
$$

where the

$$
(\hat{\eta})_{R \tilde{R}}^{j \tilde{j}}=\eta_{R}^{j} \delta_{R \tilde{R}} \delta_{j \tilde{j}}
$$

correspond to the diagonal entries $\varepsilon_{\rho_{1}}+\varepsilon_{\rho_{2}}$ of (2.1). For simplicity, we choose for the $\eta_{R}^{j}$ independent random variables with the distribution function $\rho_{0}(\eta)$. We have typically $\rho_{0}(\eta) \simeq 1 /\left(2 W_{b}\right)$ for $|\eta| \leq W_{b}$ where $W_{b}$ is twice the bandwidth of the disordered one-electron Hamiltonian. The operator $\hat{\zeta}$ corresponds to the interaction matrix elements (2.3). The matrix elements are independent Gaussian random variables with zero mean and variance

$$
\left\langle\left(\hat{\zeta}_{R \tilde{R}}^{j \tilde{j}}\right)^{2}\right\rangle=\frac{1}{2}\left(1+\delta_{R \tilde{R}} \delta_{j \tilde{j}}\right) a(|R-\tilde{R}|) v(j) v(\tilde{j})
$$

The smooth functions $a(|R-\tilde{R}|)$ and $v(j)$ decay exponentially on the scale $L_{1}$. We assume the typical behavior:

$$
a(|R-\tilde{R}|) \sim \begin{cases}U^{2} L_{1}^{-3 d} & ,|R-\tilde{R}| \lesssim L_{1} \\ U^{2} L_{1}^{-3 d} e^{-2|R-\tilde{R}| / L_{1}} & ,|R-\tilde{R}| \gg L_{1}\end{cases}
$$

and

$$
v(j) \sim \begin{cases}1 & ,|j| \lesssim L_{1} \\ e^{-4|j| / L_{1}} & ,|j| \gg L_{1}\end{cases}
$$

which is justified by Eqs. (2.3) and (2.4). The function $a(|R-\tilde{R}|)$ describes how the coupling strength decreases exponentially as the distance of the centers of mass increases, while $v(j)$ describes how the "size" of the product states reduces the coupling.

In Eqs. (2.9), (2.10), we have applied the simplified estimate (2.5) for the typical strength of the interaction coupling matrix elements. However, the presented random matrix model and its subsequent treatment is given in terms of the general functions $a(R)$ and $v(j)$. It is straightforward to use different, more "realistic", estimates instead of (2.5), e.g. to take effects of ballistic or diffusive motion for length scales $L \lesssim L_{1}$ into account.

\section{B. Nonlinear $\sigma$ Model}

Our goal is to investigate the spectral statistics, the transport and the localization properties of the ERMH (2.6) applying the supersymmetry technique [5.29]. In the following, we choose a particular realization of the diagonal elements $\eta_{R}^{j}$ and perform the ensemble 
average only with respect to $\hat{\zeta}$. This particular average is denoted by $\langle\cdots\rangle_{\zeta}$. We consider the generating functional

$$
\begin{aligned}
F(J) & =\left\langle\int D \psi \exp \left[\frac{i}{2} \bar{\psi}\left(E-\mathcal{H}+\left(\frac{\omega}{2}+i \varepsilon\right) \Lambda+J\right) \psi\right]\right\rangle_{\zeta} \\
& =\left\langle\operatorname{sdet}^{-1 / 2}\left(E-\mathcal{H}+\left(\frac{\omega}{2}+i \varepsilon\right) \Lambda+J\right)\right\rangle_{\zeta} .
\end{aligned}
$$

Here, $\psi$ is a supervector with components $\psi_{j}(R)$, which are 8-dimensional supervectors with entries $z_{1}, \bar{z}_{1}, \chi_{1}, \bar{\chi}_{1}, z_{2}, \bar{z}_{2}, \chi_{2}, \bar{\chi}_{2}$, where $z_{\nu}\left(\chi_{\nu}\right)$ are complex bosonic (fermionic) variables. The diagonal matrix $\Lambda=\operatorname{diag}\left(\mathbf{1}_{4},-\mathbf{1}_{4}\right)$ describes the grading into advanced and retarded Green's functions, $\omega$ is a frequency, $J$ is a source matrix, and $\bar{\psi}$ is given by $\bar{\psi}=\psi^{\dagger} \Lambda$. For the graded determinant ("sdet") and for the graded trace ("str") we use the convention that $\operatorname{str}(A)=\operatorname{tr}\left(A_{B B}\right)-\operatorname{tr}\left(A_{F F}\right)$ and $\operatorname{sdet}(A)=\exp (\operatorname{str} \ln (A))$, where $A_{B B}\left(A_{F F}\right)$ is the boson-boson (fermion-fermion) block of the supermatrix $A$.

Using suitable choices [29, 30] for $J$ and taking derivatives of $F(J)$ with respect to $J$ at $J=0$, one can obtain moments of the Green's function. These contain all the informations about the spectral statistics and transport properties we are interested in. Our strategy is the following: We derive a nonlinear $\sigma$ model from the functional $F(J)$ and discuss its physical implications by comparing our result with the $\sigma$ model description of noninteracting electrons derived by Efetov [5].

The ensemble average with respect to $\hat{\zeta}$ is readily performed and results in

$$
F(J)=\int D \psi \exp \left[\frac{i}{2} \bar{\psi}\left(E-\hat{\eta}+\left(\frac{\omega}{2}+i \varepsilon\right) \Lambda+J\right) \psi-\frac{1}{8} \sum_{R, \tilde{R}} a(|R-\tilde{R}|) \operatorname{str}[K(R) K(\tilde{R})]\right]
$$

where $K(R)$ are $8 \times 8$ supermatrices given by

$$
K(R)=\sum_{j} v(j) \psi_{j}(R) \bar{\psi}_{j}(R)
$$

We see that the statistical properties (2.8) of $\hat{\zeta}$ allow for a convenient decoupling into the center of mass coordinates $R$ and the relative coordinates $j$. The quartic term in (2.12) is decoupled in a standard way [5,29] by a Hubbard-Stratonovich transformation. This introduces an integral over a field of $8 \times 8$ supermatrices $\sigma(R)$ with the same symmetries as $K(R)$, and we get

$$
\begin{aligned}
& F(J)=\int D \sigma \exp \left(-\mathcal{L}_{1}[\sigma]\right), \\
& \tilde{\mathcal{L}}_{2}[\sigma]=\frac{1}{8} \sum_{R, \tilde{R}}\left(A^{-1}\right)_{R \tilde{R}} \operatorname{str}[\sigma(R) \sigma(\tilde{R})]+\frac{1}{2} \operatorname{str}_{(j R)} \ln \left(E-\hat{\eta}+\left(\frac{\omega}{2}+i \varepsilon\right) \Lambda+J+\frac{1}{2} \hat{\sigma}\right)
\end{aligned}
$$

Here, $A^{-1}$ is the inverse of the matrix $A_{R \tilde{R}}=a(|R-\tilde{R}|)$ and $\hat{\sigma}$ is given by

$$
(\hat{\sigma})_{R \tilde{R}}^{j \tilde{j}}=v(j) \sigma(R) \delta_{R \tilde{R}} \delta_{j \tilde{j}}
$$


The supertrace (" $\operatorname{str}_{(j R)}$ ") appearing in (2.14) extends over the full $8 L^{2 d}$-dimensional supervector space, where $L$ is the system size assumed to be much larger than $L_{1}$. To proceed, we apply as usual a saddle point approximation, which is valid in the limit $L_{1} \gg 1$. The corresponding saddle point equations $\delta \mathcal{L}_{1} / \delta \sigma(R)=0$ can be written in the form (for $J=0$ and $\omega=0)$

$$
\sigma(R)=-\sum_{\tilde{R}} a(|R-\tilde{R}|) \sum_{j} v(j) \frac{1}{E-\eta_{\tilde{R}}^{j}+i \varepsilon \Lambda+\frac{1}{2} v(j) \sigma(\tilde{R})} .
$$

The sums extend over $L_{1}^{2 d}$ (contributing) terms and, applying a central limit theorem argument, we may replace the particular values of $\eta_{\tilde{R}}^{j}$ by an average with respect to the distribution $\rho_{0}(\eta)$ (see below for a more precise justification). Then (2.16) can be solved by a homogeneous (i.e. $R$-independent) ansatz $\sigma(R)=\Gamma_{0}+i \Gamma_{1} \Lambda$, where $\Gamma_{0}, \Gamma_{1}$ are determined by the implicit equation

$$
\begin{aligned}
\Gamma_{0}+i \Gamma_{1} \Lambda & =-B_{0} \sum_{j} v(j) \int d \eta \rho_{0}(\eta) \frac{1}{E-\eta+i \varepsilon \Lambda+\frac{1}{2} v(j)\left(\Gamma_{0}+i \Gamma_{1} \Lambda\right)}, \\
B_{0} & =\sum_{R} a(|R|) .
\end{aligned}
$$

In the limit $L_{1} \gg 1, \Gamma_{0}$ and $\Gamma_{1}$ are much smaller than the typical integration range $2 W_{b}$ for $\eta$ and we find

$$
\begin{aligned}
& \Gamma_{0} \simeq-B_{0} S_{1} \mathcal{P} \int d \eta \rho_{0}(\eta) \frac{1}{E-\eta}, \\
& \Gamma_{1} \simeq \pi B_{0} S_{1} \rho_{0}(E)
\end{aligned}
$$

with $S_{\nu}=\sum_{j}[v(j)]^{\nu} \sim L_{1}^{d}$ and $\mathcal{P} \int d \eta(\cdots)$ a principal value integral. From Eqs. (2.9) and (2.10) we find the estimate

$$
\Gamma_{1} \sim \frac{U^{2}}{W_{b}} L_{1}^{-d} \ll W_{b}
$$

if $U$ and $W_{b}$ are of the same order of magnitude. The imaginary part of the r.h.s. of (2.16) is a sum of Lorentzians with a typical width $\Gamma_{1} v(j)$. The most important contributions in this sum arise from $|j| \lesssim L_{1}$ and $|R-\tilde{R}| \lesssim L_{1}$ such that $v(j) \sim 1$. The maxima $\eta_{R}^{j}$ of the Lorentzians have thus a typical distance $\sim 2 W_{b} L_{1}^{-2 d} \ll \Gamma_{1}$ and we are in the regime of well overlapping resonances. Therefore it is indeed allowed to replace (2.16) by its average with respect to $\eta_{\tilde{R}}^{j}$. As usual, the saddle point $\Gamma_{0}+i \Gamma_{1} \Lambda$ is not unique and we have to consider a complete saddle point manifold $\Gamma_{0}+i \Gamma_{1} Q$ with $Q=T^{-1} \Lambda T, T$ being an element of a certain supermatrix group [5.29]. The supermatrix $Q$ fulfills the nonlinear constraint $Q^{2}=1$ and is in our case a member of the orthogonal $\sigma$ model space. In the following, we replace $\sigma(R)$ by $\Gamma_{0}+i \Gamma_{1} Q(R)$ where each $Q(R)$ fulfills this constraint but may slowly vary in $R$-space. The matrix $A_{R \tilde{R}}=a(|R-\tilde{R}|)$ has eigenvalues $\tilde{a}(k)=\sum_{R} e^{i k R} a(|R|)$ and the eigenvalues of $A^{-1}$ are just given by

$$
\frac{1}{\tilde{a}(k)} \simeq B_{0}+k^{2} \frac{B_{2}}{B_{0}^{2}} \quad, \quad B_{2}=\frac{1}{2 d} \sum_{R} R^{2} a(|R|) \quad .
$$


Here we have applied an expansion with respect to small momenta $k$ since mainly the slow modes will give the relevant contributions [5,31]. In analogy to [31, we evaluate the first term of the action (2.14) by performing a Fourier transformation, applying (2.22) and afterwards transforming back to position space. Since $Q(R)$ varies slowly with $R$, we may go over to a continuum limit and obtain the following expression for the functional $F(J)$ :

$$
\begin{aligned}
F(J) & =\int D Q \exp \left(-\mathcal{L}_{2}[Q]\right) \\
\mathcal{L}_{2}[Q] & =-\frac{\Gamma_{1}^{2} B_{2}}{8 B_{0}^{2}} \int d R \operatorname{str}\left(\left[\nabla_{R} Q(R)\right]^{2}\right)+\frac{1}{2} \operatorname{str}_{(j R)} \ln \left(E-\hat{\eta}+\left(\frac{\omega}{2}+i \varepsilon\right) \Lambda+J+\frac{1}{2} \hat{\sigma}\right) \\
(\hat{\sigma})_{R \tilde{R}}^{j \tilde{j}} & =v(j)\left[\Gamma_{0}+i \Gamma_{1} Q(R)\right] \delta_{R \tilde{R}} \delta_{j \tilde{j}}
\end{aligned}
$$

For convenience we have kept the discrete notation for $R$ in the second term of the action $\mathcal{L}_{2}$. Expression (2.23) defines the nonlinear $\sigma$ model suitable to describe the ERMH.

\section{Breit-Wigner Form for the Local Density of States}

As a first application, we determine the local density of states,

$$
\rho_{R}^{j}(E)=-\frac{1}{\pi} \lim _{\varepsilon \rightarrow 0+} \operatorname{Im}\left\langle\left\langle R j\left|(E-\mathcal{H}+i \varepsilon)^{-1}\right| R j\right\rangle\right\rangle_{\zeta}
$$

This quantity can be calculated using small $4 \times 4$ supermatrices $\sigma(R)$ in (2.14) and replacing $\Lambda$ by 1 . Then only the saddle point $\Gamma_{0}+i \Gamma_{1}$ (and not a full saddle point manifold) contributes. Choosing for $J$ a supermatrix that has its only nonvanishing diagonal entry $x L_{g}$ at site $(j, R)$ ( $L_{g}$ is a diagonal supermatrix with entries +1 and -1 in the boson-boson and fermionfermion blocks, respectively), one finds

$$
\rho_{R}^{j}(E)=\frac{1}{\pi} \frac{\frac{1}{2} \Gamma_{1} v(j)}{\left[E-\eta_{R}^{j}+\frac{1}{2} \Gamma_{0} v(j)\right]^{2}+\left[\frac{1}{2} \Gamma_{1} v(j)\right]^{2}}
$$

This is a Breit-Wigner function with the width $\frac{1}{2} \Gamma_{1} v(j)$. The same result can also be obtained by a standard diagrammatic expansion in powers of $\hat{\zeta}$. The complex self-energy is then given by by $\Sigma_{R}^{j}=-\frac{1}{2}\left(\Gamma_{0}+i \Gamma_{1}\right) v(j)$ with $\Gamma_{0}$ and $\Gamma_{1}$ determined by (2.17) and (2.18).

To interpret (2.25) we note that the noninteracting product states with eigenenergies $\eta_{R}^{j}$ acquire a finite width $\Gamma_{1} v(j)$ due to the interaction. This width is of the order $\Gamma_{1}$ for $|j| \leq L_{1}$ and decreases esponentially as $\Gamma_{1} e^{-4|j| / L_{1}}$ for $|j| \gg L_{1}$. This means that those states $\eta_{R}^{j}$ constructed from well separated one-electron states $\left(|j| \gg L_{1}\right)$ constitute good "quasi-levels" with exponentially large life times. We may consider a critical pair size $L_{c}$ such that the pair states with $2|j| \lesssim L_{c}$ in a system of size $L_{\text {sys }}$ are still reasonably well

coupled. For this, the effective level spacing $\Delta_{\text {eff }}=W_{b}\left(L_{c} L_{\text {sys }}\right)^{-d}$ has to be compared with the smallest possible level width $\Gamma_{1} e^{-2 L_{c} / L_{1}}$. This yields the rough estimate $L_{c} \sim L_{1} \ln L_{\text {sys }}$. The $\eta_{R}^{j}$ with $|j|>L_{c}$ contribute to a discrete point spectrum whereas the states with $|j| \lesssim L_{c}$ are well mixed and form effective pairs of maximal size $L_{c} \sim L_{1} \ln L_{\text {sys }}$. 


\section{TIP-conductance $g_{2}$ at scale $L>L_{1}$}

We now want to describe the dynamics of these pairs in terms of the effective $\sigma$ model (2.23) with the action $\mathcal{L}_{2}[Q]$. In order to understand the relation with a disordered metal, we recall a few results for the corresponding $\sigma$ model description given by Efetov [5] and characterized by the action

$$
\mathcal{L}_{\text {met }}[Q]=-\frac{\pi}{8} \nu \mathcal{D} \int d r \operatorname{str}\left((\nabla Q)^{2}\right)+\frac{\pi \nu}{2}\left(-i \frac{\omega}{2}+\varepsilon\right) \int d r \operatorname{str}(Q(r) \Lambda)
$$

Here $\mathcal{D}$ denotes the classical diffusion constant and $\nu$ the local density of states. Efetov derived [5] this action for frequencies $|\omega| \ll 1 / \tau$ where $\tau$ is the elastic scattering time due to the disorder. The action $\mathcal{L}_{\text {met }}[Q]$ therefore describes the quantum diffusion for time scales larger than $\tau$. The nontrivial quantum properties are due to the nonlinear constraint $Q^{2}=1$ of the supermatrix $Q(r)$. One may parameterize [5] $Q$ by $Q=\Lambda \frac{1+i P}{1-i P}$ where $P$ is a supermatrix with only offdiagonal entries in the $\Lambda$-grading, $\Lambda P \Lambda=-P$. Expanding the action (2.26) with respect to $P$, Efetov showed that a perturbative evaluation of the $\sigma$ model is completely equivalent to the diagrammatic theory of disordered metals in terms of (interacting) Diffuson and Cooperon modes. The leading order corresponds to the classical diffusion and the higher order terms yield the first quantum corrections. Furthermore, Efetov found that the action (2.26) leads to the well known level statistics 32 of random matrix theory if the considered range of energies is smaller than the Thouless energy $E_{c}=\mathcal{D} / L^{2}$ (i.e. $\left.|\omega| \ll E_{c}\right)$. For frequencies $\omega$ such that $E_{c} \ll|\omega| \ll 1 / \tau$ and in the metallic regime $E_{c} \gg \Delta$ ( $\Delta$ denotes the level spacing), the corrections to the universal Wigner-Dyson statistics have been calculated by Altshuler and Shklovskii [33]. In one dimension, the $\sigma$ model (2.26) describes a (thick) wire. All the wave functions are localized [8,5] with a localization length $\xi=2 \pi \nu \mathcal{D}$ (for $L \gg \xi$ ).

Comparing the first terms of the actions of (2.23) and (2.26), one gets for two interacting particles

$$
\nu_{\mathrm{eff}} \mathcal{D}_{\mathrm{eff}}=\frac{1}{\pi} \frac{\Gamma_{1}^{2} B_{2}}{B_{0}^{2}}=\pi \rho_{0}(E)^{2} S_{1}^{2} B_{2} \sim \frac{U^{2}}{W_{b}^{2}} L_{1}^{2},
$$

where the second identity results from Eq. (2.20). We have introduced an effective (pair) diffusion constant $\mathcal{D}_{\text {eff }}$ and an effective local density of states $\nu_{\text {eff }}$. For (quasi) one-dimensional systems the quantity in (2.27) is proportional to the localization length $L_{2}$ for the center of mass coordinate for the pairs and we recover Shepelyansky's result [16]: $L_{2} \sim\left(U^{2} / W_{b}^{2}\right) L_{1}^{2}$. We reiterate that we have taken all, also the weakly coupled, product states into account. It has been suggested [26] that these weakly coupled states lead to a logarithmic correction, $L_{2} \sim L_{1}^{2} / \ln L_{1}$. From our analytical considerations we find no evidence for such a behavior.

In order to extract the diffusion constant $\mathcal{D}_{\text {eff }}$ from (2.27), we still need a reasonable estimate for the effective local density of (product) states $\nu_{\text {eff }}$ that contribute to the diffusion. Naively, one could consider the effective size $L_{c} \sim L_{1} \ln L_{\text {sys }}$ of the pair states, which we estimated in the above discussion subsequent to Eq. (2.25). This would yield the estimates

$$
\nu_{\mathrm{eff}} \sim \rho_{0}(E) L_{c}^{d} \sim \rho_{0}(E) L_{1}^{d}\left(\ln L_{\mathrm{sys}}\right)^{d} \Rightarrow \mathcal{D}_{\mathrm{eff}} \sim \frac{U^{2}}{W_{b}} L_{1}^{2-d}\left(\ln L_{\mathrm{sys}}\right)^{-d}
$$


However, this simple picture assumes that all product states up to a size of $L_{c}$ contribute equally to the diffusion. Obviously, this is not the case. Similarly to the frequency condition $|\omega|<1 / \tau$ for a disordered metal, we have to require $|\omega|<1 / \tau_{c}$ to justify (2.28), where $1 / \tau_{c}=\Gamma_{1} v\left(L_{c}\right)=\Delta_{\text {eff }}$ is the effective level spacing of the well coupled product states, which is by definition equal to the inverse life time of pairs of size $L_{c}$. We recall that $1 / \tau_{c}=W_{b} L_{c}^{-2 d} \sim W_{b}\left(L_{1} \ln L_{\mathrm{sys}}\right)^{-2 d}$ is much smaller than the energy scale $\Gamma_{1} \sim\left(U^{2} / W_{b}\right) L_{1}^{-d}$, which is the inverse life time of the best coupled states (of size $|j| \lesssim L_{1}=L_{c} / \ln L_{\text {sys }}$ ). For frequencies between $1 / \tau_{c}$ and $\Gamma_{1}$, we thus expect that the number $N(\omega)$ of contributing states depends on $\omega$ via:

$$
N(\omega)=\left[L_{\mathrm{eff}}(\omega)\right]^{d} \quad, \quad L_{\mathrm{eff}}(\omega)=\frac{L_{1}}{4} \ln \left(\frac{\Gamma_{1}}{|\omega|}\right),
$$

where $L_{\mathrm{eff}}(\omega)$ is the pair size such that the corresponding inverse life time $\Gamma_{1} v\left(L_{\mathrm{eff}}(\omega)\right)$ equals $|\omega|$. Eq. (2.29) results in a frequency dependent density of states and diffusion constant,

$$
\nu_{\mathrm{eff}}(\omega) \sim \rho_{0}(E)\left(L_{1} \ln \left(\Gamma_{1} /|\omega|\right)\right)^{d} \Rightarrow \mathcal{D}_{\mathrm{eff}}(\omega) \sim \frac{U^{2}}{W_{b}} L_{1}^{2-d}\left(\ln \left(\Gamma_{1} /|\omega|\right)\right)^{-d} .
$$

This estimate is valid for $1 / \tau_{c} \lesssim|\omega| \lesssim \Gamma_{1}$, whereas for $|\omega| \lesssim 1 / \tau_{c}$ Eq. (2.28) applies. The estimates (2.28) and (2.30), based on the simple but physical life time argument, can also be obtained in a more formal way directly from the $\sigma$ model action (2.23). For this we rewrite $\mathcal{L}_{2}[Q]$ (at vanishing source term $J=0$ ) as

$$
\mathcal{L}_{2}[Q]=-\frac{\pi}{8} \nu_{\text {eff }} \mathcal{D}_{\text {eff }} \int d R \operatorname{str}\left((\nabla Q)^{2}\right)+\int d R f_{R}(Q(R))
$$

where

$$
f_{R}(Q)=\frac{1}{2} \sum_{j} \operatorname{str} \ln \left(E-\eta_{R}^{j}+\left(\frac{\omega}{2}+i \varepsilon\right) \Lambda+\frac{1}{2} v(j)\left(\Gamma_{0}+i \Gamma_{1} Q\right)\right)
$$

is some effective $Q$-potential for the $\sigma$ model. In Appendix $\mathrm{A}$, this expression for the effective potential is simplified to give

$$
f_{R}(Q) \simeq-i \frac{\pi}{4} \omega h\left(\frac{\Gamma_{1}}{\omega}\right) \rho_{0}(E) \operatorname{str}(Q \Lambda),
$$

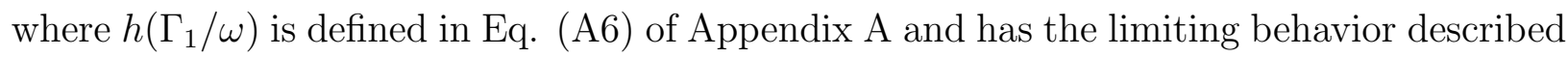
in (A7 A9). Comparing (2.33) with the second term in the action (2.26), we obtain a complex effective density of states,

$$
\nu_{\mathrm{eff}}(\omega)=\rho_{0}(E) h\left(\frac{\Gamma_{1}}{\omega}\right) .
$$

For the two cases ( $(\sqrt{\mathrm{A} 8})$ and ( $(\mathrm{A9})$ we directly recover the estimates (2.30) and (2.28), respectively. On the other hand, the frequency range $|\omega| \gg \Gamma_{1}$ correponds to

$$
f_{R}(Q) \simeq \frac{\pi}{4} \Gamma_{1} \operatorname{str}(Q \Lambda)
$$


which means that the action $\mathcal{L}_{2}[Q]$ no longer depends on $\omega$.

To summarize, we have found that for frequencies smaller than $\Gamma_{1}$, the $\sigma$ model action $\mathcal{L}_{2}[Q]$ can be written in a similar form as the action $\mathcal{L}_{\text {met }}[Q]$ for a disordered metal,

$$
\mathcal{L}_{2}[Q]=-\frac{\pi}{8} \nu_{\text {eff }}(\omega) \mathcal{D}_{\text {eff }}(\omega) \int d r \operatorname{str}\left((\nabla Q)^{2}\right)+\frac{\pi \nu_{\text {eff }}(\omega)}{2}\left(-i \frac{\omega}{2}+\varepsilon\right) \int d r \operatorname{str}(Q(r) \Lambda)
$$

It was necessary to introduce both a frequency dependent diffusion constant $\mathcal{D}_{\text {eff }}(\omega)$ and a frequency dependent density of states $\nu_{\text {eff }}(\omega)$,

$$
\begin{aligned}
& \mathcal{D}_{\text {eff }}(\omega) \sim \begin{cases}\frac{U^{2}}{W_{b}} L_{1}^{2-d}\left(\ln L_{\text {sys }}\right)^{-d}, & |\omega| \lesssim 1 / \tau_{c} \\
\frac{U^{2}}{W_{b}} L_{1}^{2-d}\left(\ln \left(\frac{\Gamma_{1}}{|\omega|}\right)\right)^{-d}, & 1 / \tau_{c} \lesssim|\omega| \ll \Gamma_{1}\end{cases} \\
& \nu_{\text {eff }}(\omega)=\frac{\sigma_{\text {eff }}}{\mathcal{D}_{\text {eff }}(\omega)}, \quad \sigma_{\text {eff }}=\frac{\Gamma_{1}^{2} B_{2}}{\pi B_{0}^{2}} \sim \frac{U^{2}}{W_{b}^{2}} L_{1}^{2} .
\end{aligned}
$$

Here, we have defined in analogy to a disordered metal via the Einstein relation a "pair conductivity" $\sigma_{\text {eff }}$ which does not depend on frequency. This determines the TIP-conductance $g_{2}$ mentioned in the introduction through the usual relation between conductance and conductivity: $g_{2}=\sigma_{\text {eff }} L_{\text {sys }}^{d-2}$. The analogy with a disordered metal allows us to draw some very interesting and far reaching conclusions. First, we can identify the coupling constant $\frac{\pi}{8} \nu_{\text {eff }} \mathcal{D}_{\text {eff }}=\frac{\pi}{8} \sigma_{\text {eff }}$ in $\mathcal{L}_{2}[Q]$ as a universal scaling parameter. The corresponding scaling function is precisely the same as that of a disordered metal provided the latter is described by the "standard" $\sigma$ model (2.26). In particular, the perturbative evaluation of the $\beta$-function in $2+\varepsilon$ dimensions [5] is also valid for our problem of diffusing or localized electron pairs (the second term in $\mathcal{L}_{2}[Q]$ containing the supertrace $\operatorname{str}(Q \Lambda)$ is not affected under the renormalization [5]). In one dimension, we are able to directly identify the pair localization length $L_{2} \sim \sigma_{\text {eff }} \sim\left(U^{2} / W_{b}^{2}\right) L_{1}^{2}$. These remarks rigorously justify Imry's application [17] of the Thouless scaling picture [1].

\section{E. TIP-Dynamics for $L_{1}<<L \leq L_{2}$}

A second conclusion concerns the pair-dynamics in the metallic (or diffusive) regime ( $L \lesssim L_{2}$ for $d=1,2$ ) where the $\sigma$ model can be treated perturbatively (cp. Ref. [5]). The relevant diffusion propagator $\mathcal{R}(q, \omega)=\left[\sigma_{\text {eff }} q^{2}-i \omega \nu_{\text {eff }}(\omega)\right]^{-1}=\left\{\nu_{\text {eff }}(\omega)\left[\mathcal{D}_{\text {eff }}(\omega) q^{2}-i \omega\right]\right\}^{-1}$ now contains the frequency dependent diffusion constant (2.37) and density of states (2.38). This gives rise to some subtle modifications of standard diffusion. The precise calculation of the diffusion propagator $\tilde{\mathcal{R}}(R, t)=(2 \pi)^{-(d+1)} \int d \omega \int d q \mathcal{R}(q, \omega) e^{i(q R-\omega t)}$ in position and time space seems to be rather difficult due to the various frequency dependencies involved. However, in a qualitative approximation, we may replace $\omega$ by $1 / t$. This shows that the pairs propagate according to

$$
\left\langle R^{2}(t)\right\rangle \sim \mathcal{D}_{\mathrm{eff}}(1 / t) t \sim \begin{cases}L_{1}^{2}\left(1+\alpha \Gamma_{1} t+\cdots\right)=L_{1}^{2}+\tilde{\alpha} D_{0} t+\cdots & , t \ll \Gamma_{1}^{-1} \\ D_{0}\left[\ln \left(\Gamma_{1} t\right)\right]^{-d} t & , \Gamma_{1}^{-1} \ll t \lesssim \tau_{c} \\ D_{0}\left[\ln L_{\mathrm{sys}}\right]^{-d} t & , \tau_{c} \lesssim t\end{cases}
$$


We have used (see 2.37) the notation $D_{0}=\left(U^{2} / W_{b}\right) L_{1}^{2-d}$, and $\alpha$ and $\tilde{\alpha}$ are numerical constants of the order of unity. For very small time scales $t \ll \Gamma_{1}^{-1}$ the dynamics is not diffusive. The corresponding estimate given in (2.39) for these time scales may formally be obtained by a naive combination of (A7), (2.34) and (2.38), which is strictly speaking not justfied. However, a more sophisticated analysis for the classical propagator $\tilde{\mathcal{R}}(R, t)$ shows that this estimate is indeed correct. The physical interpretation of (2.39) is that the electron pairs are immediately (on time scales which are not resolved by the ERMH) delocalized on a scale $L_{1}$, due to interaction induced random hoppings. Then a diffusion with diffusion constant $D_{0}$ sets in. Eventually the diffusion constant is suppressed by the time dependent factor $\left[\ln \left(\Gamma_{1} t\right)\right]^{-d}$ which saturates at $\left[\ln L_{\mathrm{sys}}\right]^{-d}$ for $t \gtrsim \tau_{c}$.

We also expect that, due to the frequency dependence of $\nu_{\text {eff }}(\omega)$, the number of diffusing states depends on time as $N(1 / t)$ with $N(\omega)$ defined in (2.29). The time dependent pair size therefore behaves as $L_{1} \ln \left(\Gamma_{1} t\right)$ for $\Gamma_{1}^{-1} \ll t \lesssim \tau_{c}$ and as $L_{1} \ln L_{\text {sys }}$ for $\tau_{c} \lesssim t$. In recent numerical simulations [21,22] of two "interacting" kicked rotators, Borgonovi and Shepelyansky indeed observed the logarithmic increase of the pair size with time.

As a further illustration, we consider the probability that an electron pair performs a transition from a product state $\mid R j>$ to another state $\mid \tilde{R} \tilde{j}>$ in the time $t>0$. This probability is given by

$$
|<\tilde{R} \tilde{j}| e^{-i \mathcal{H} t}|R j>|^{2}=\frac{1}{4 \pi^{2}} \int d E \int d \omega e^{-i \omega t}\left(G_{\tilde{R} R}^{\tilde{j} j}\right)^{(+)}\left(E+\frac{\omega}{2}\right)\left(G_{R \tilde{R}}^{j \tilde{j}}\right)^{(-)}\left(E-\frac{\omega}{2}\right),
$$

where

$$
\left(G_{\tilde{R} R}^{\tilde{j} j}\right)^{( \pm)}(E)=<\tilde{R} \tilde{j}\left|\frac{1}{E \pm i \varepsilon-\mathcal{H}}\right| R j>
$$

denotes the matrix elements of the advanced or retarded Green's function. The ensemble average with respect to $\hat{\zeta}$ of the transition probability can be calculated from the functional $F(J)$ (choosing a suitable source matrix $J$ and taking the derivatives). Omitting technical details, we give the result of a perturbative evaluation in the diffusive regime using the $\sigma$ model (2.36):

$$
\begin{aligned}
\left\langle\left|\left\langle\tilde{R} \tilde{j}\left|e^{-i \mathcal{H} t}\right| R j\right\rangle\right|^{2}\right\rangle_{\zeta} \sim & \int d E \int d \omega e^{-i \omega t} \\
& \times\left(\frac{\frac{1}{2 \pi} \Gamma_{1} v(\tilde{j})}{\left(E-\eta_{\tilde{R}}^{\tilde{j}}+\frac{1}{2} \Gamma_{0} v(\tilde{j})\right)^{2}+\left(\frac{1}{2} \Gamma_{1} v(\tilde{j})-\frac{i}{2} \omega\right)^{2}}\right) \\
& \times\left(\frac{\frac{1}{2 \pi} \Gamma_{1} v(j)}{\left(E-\eta_{R}^{j}+\frac{1}{2} \Gamma_{0} v(j)\right)^{2}+\left(\frac{1}{2} \Gamma_{1} v(j)-\frac{i}{2} \omega\right)^{2}}\right) \\
& \times(2 \pi)^{-d} \int d q \mathcal{R}(q, \omega) e^{i q(R-\tilde{R})} .
\end{aligned}
$$

The contribution in the last line is the "diffusion" propagator in position and frequency space with $\mathcal{R}(q, \omega)=\left[\sigma_{\text {eff }} q^{2}-i \omega \nu_{\text {eff }}(\omega)\right]^{-1}$ as above. Physically, it describes how the electron pair diffuses along its center of mass coordinate $R$ [with the modifications shown in 
(2.39)]. The only information about the relative coordinate $j$ is contained in the Lorentzian prefactors in (2.39). These effectively define a resonance condition for the diagonal energies: $\left|\eta_{R}^{j}-\eta_{\tilde{R}}^{\tilde{j}}\right| \lesssim \Gamma_{1} \min [v(j), v(\tilde{j})]$. For the well coupled product states $(v(j) \sim 1, v(\tilde{j}) \sim 1)$, we recover that essentially states inside a band of width $\Gamma_{1}$ are coupled by the diffusive transport processes. For the badly coupled product states $\left(v(j) \sim e^{-4|j| / L_{1}}, v(\tilde{j}) \sim e^{-4|\tilde{j}| / L_{1}}\right.$, with $\left.|j|,|\tilde{j}| \gg L_{1}\right)$ this resonance condition is practically never fulfilled and the transition probability becomes exponentially small due to the Lorentzian factors. However, for certain improbable realizations of the $\eta_{R}^{j}$ the resonance condition may be fulfilled even for two badly coupled product states. Then the corresponding transition probability experiences a strong enhancement.

\section{F. TIP Level Statistics at $L>L_{1}$}

The $\sigma$ model action (2.36) also provides a very convenient tool to discuss level correlations. Actually, the two point correlation function, defined by

$$
Y_{2}(\omega)=\frac{1}{\langle\rho(E)\rangle\langle\rho(E+\omega)\rangle}(\langle\rho(E)\rangle\langle\rho(E+\omega)\rangle-\langle\rho(E) \rho(E+\omega)\rangle),
$$

$(\rho(E)$ is the unaveraged total density of states) can be calculated as in Ref. [5]. Of course, in this way, we only obtain the correlations of the well coupled (diffusing) pair states. The isolated pair states are mainly uncorrelated with the well coupled states and among themselves. Therefore, their contribution to the correlation function (2.43) essentially cancels out. For a finite system of size $L$, the diffusive dynamics defines an energy scale $E_{c}^{(2)}$, the "pair Thouless energy", which is in our case determined by the implicit equation $\mathcal{D}_{\text {eff }}\left(E_{c}^{(2)}\right) / L^{2}=E_{c}^{(2)}$. For frequencies smaller than $E_{c}^{(2)}$ the second term of the action $\mathcal{L}_{2}[Q]$ in (2.36) dominates the level correlations. $Y_{2}(\omega)$ can exactly be expressed by the same integral (over one $Q$-matrix) as in [5]. Therefore we recover the random matrix result

$$
Y_{2}(\omega)=Y_{2}^{(G O E)}\left(\frac{\omega}{\Delta(\omega)}\right) \quad, \quad \Delta(\omega)^{-1}=L^{d} \nu_{\mathrm{eff}}(\omega) \quad, \quad|\omega| \ll E_{c}^{(2)}
$$

with $\nu_{\text {eff }}$ as in (2.37), (2.38). $\Delta(\omega)$ is a frequency dependent effective levelspacing and $Y_{2}^{(G O E)}(r)$ is the universal spectral correlation function of the Gaussian orthogonal ensemble ( $r$ is the energy difference measured in units of the level spacing). For higher frequencies, i.e. $E_{c}^{(2)} \ll|\omega| \ll \Gamma_{1}$ the first (kinetic) term of $\mathcal{L}_{2}[Q]$ has to be taken into account. The perturbative evaluation of $Y_{2}(\omega)$ is completely equivalent to the diagrammatical calculation of $Y_{2}(\omega)$ given by Alt'shuler and Shklovskii [33. We get

$$
Y_{2}(\omega) \sim \frac{1}{\omega^{2}}\left(\frac{\omega}{\mathcal{D}_{\text {eff }}(\omega) / L^{2}}\right)^{d / 2} \quad, \quad E_{c}^{(2)} \ll|\omega| \ll \Gamma_{1} .
$$

Here $\mathcal{D}_{\text {eff }}(\omega) / L^{2}$ can be viewed as a frequency dependent Thouless energy that sets the scale for the level correlations in the diffusive regime.

In summary, we state that the field theoretical $\sigma$ model formulation of the effective random matrix model enabled us to recover many well known results for the localization problem of noninteracting particles. 


\section{TIGHTLY BOUND PARTICLES IN ONE DIMENSION}

In this section, we are concerned with a particular example in one dimension which allows for a more microscopic treatment than the mapping onto the effective random matrix model. Our aim is to show that the physical implications and results found in the last section are not restricted to the random matrix approach and are indeed generic for the two interacting particle problem. Moreover, in the present case we are able to generalize our treatment to more than two particles.

The essential idea is to compare the microscopic formulation of the problem of two interacting particles in a $1 \mathrm{~d}$ random potential with the related problem of independent particles in a quasi 1d disordered strip. To this end we start with the case of two interacting particles and consider the Hamiltonian

$$
H=-\frac{1}{2}\left(\partial_{x_{1}}^{2}+\partial_{x_{2}}^{2}\right)+V\left(x_{1}\right)+V\left(x_{2}\right)+U\left(\left|x_{1}-x_{2}\right|\right)
$$

where $x_{1}$ and $x_{2}$ are the coordinates of the two particles, $U\left(\left|x_{1}-x_{2}\right|\right)$ is the interaction potential, and $V(x)$ the 1 d random potential with

$$
\begin{aligned}
\langle V(x)\rangle & =0 \\
\left\langle V(x) V\left(x^{\prime}\right)\right\rangle & =c_{1} \delta\left(x-x^{\prime}\right) .
\end{aligned}
$$

Introducing new coordinates $x=\left(x_{1}+x_{2}\right) / 2$ and $y=x_{1}-x_{2}$ we have

$$
H=-\frac{1}{4} \partial_{x}^{2}+\frac{1}{2} H_{T}(y)+V(x+y / 2)+V(x-y / 2)
$$

with the transverse Hamiltonian

$$
H_{T}(y)=-2 \partial_{y}^{2}+2 U(|y|)
$$

For later convenience we define a rescaled Hamiltonian $\tilde{H}=2 H$. We will study the transport properties of $H$ at the two-particle energy $E$ (i.e., at $\tilde{E}=2 E$ for $\tilde{H}$ ) and define a one-particle reference momentum $k_{F}$ by $E=2 k_{F}^{2} / 2$. Next, we diagonalize the transverse Hamiltonian,

$$
H_{T}(y) \phi_{n}(y)=\left(-2 \partial_{y}^{2}+2 U(|y|) \phi_{n}(y)=\epsilon_{n} \phi_{n}(y)\right.
$$

where the $\phi_{n}(y)$ are real eigenfunctions and we restrict ourselves to the even case, i.e. $\phi_{n}(y)=\phi_{n}(-y)$. Similar to Dorokhov [24] we assume an attractive potential between the two particles. In particular we consider a bag model, where $U(|y|)=\infty$ for $|y|>L$ and $U(|y|)=0$ otherwise. For this example the transverse eigenfunction system is simply given by

$$
\begin{aligned}
\phi_{n}(y) & =\frac{1}{\sqrt{L}} \cos \left(k_{n} y\right), \\
k_{n} & =\frac{\pi}{L}\left(n+\frac{1}{2}\right) \quad(n=0,1,2, \ldots) \\
\epsilon_{n} & =2 k_{n}^{2}=2\left(\frac{\pi}{L}\right)^{2}\left(n+\frac{1}{2}\right)^{2} .
\end{aligned}
$$


For the case of two electrons in a random potential an attractive interaction is of course unrealistic. However, analytical arguments [16,17,23] and in particular numerical studies based on the transfer matrix approach [16,18 indicate that the sign of the interaction is basically immaterial for the transport properties of the system. In [18] no essential difference between a repulsive Hubbard-type interaction and an attractive bag interaction was found. Therefore we feel justified to proceed with the conceptually much simpler case of an attractive bag. Also, one might think of a particle pair which is indeed (tightly) bound, e.g. an exciton.

We project $\tilde{H}$ on the transverse eigenfunction system,

$$
\begin{aligned}
\tilde{H}_{n m} & =\left\langle\phi_{n}|\tilde{H}| \phi_{m}\right\rangle=\left[-\frac{1}{2} \partial_{x}^{2}+\epsilon_{n}\right] \delta_{n m}+\hat{V}_{n m}(x), \\
\hat{V}_{n m}(x) & =2 \int_{-L}^{L} d y \phi_{n}(y) \phi_{m}(y)[V(x+y / 2)+V(x-y / 2)] .
\end{aligned}
$$

Thus we have mapped the problem on a 1d, many channel Hamiltonian. We define a diagonal matrix of longitudinal momenta,

$$
\kappa=\operatorname{diag}\left(\kappa_{1} \ldots \kappa_{n} \ldots\right) ; \quad \kappa_{n}=\sqrt{2\left(\tilde{E}-\epsilon_{n}\right)}
$$

The $N$ open channels of the problem are defined by the condition $k_{n}=\sqrt{\epsilon_{n} / 2}<k_{F}$.

\section{A. Mapping onto a Quasi-1d Transfer Matrix Approach Without Interaction}

For comparison, let us now consider one electron in a disordered $2 \mathrm{~d}$ strip,

$$
H=-\frac{1}{2}\left(\partial_{x_{1}}^{2}+\partial_{x_{2}}^{2}\right)+U\left(\left|x_{2}\right|\right)+V\left(x_{1}, x_{2}\right) \quad .
$$

Here, $x_{1}$ and $x_{2}$ are the two space coordinates, $U\left(\left|x_{2}\right|\right)$ is the confining potential defining the strip and $V\left(x_{1}, x_{2}\right)$ the $2 \mathrm{~d}$ random potential with the statistical properties

$$
\begin{aligned}
\left\langle V\left(x_{1}, x_{2}\right)\right\rangle & =0 \\
\left\langle V\left(x_{1}, x_{2}\right) V\left(x_{1}^{\prime}, x_{2}^{\prime}\right)\right\rangle & =c_{2} \delta\left(x_{1}-x_{1}^{\prime}\right) \delta\left(x_{2}-x_{2}^{\prime}\right)
\end{aligned}
$$

For notational simplicity we do not explicitly distinguish between the two cases considered here and hope that no confusion will arise. In close analogy to the previous problem of two particles we diagonalize the transverse Hamiltonian

$$
H_{T}\left(x_{2}\right)=-\frac{1}{2} \partial_{x_{2}}^{2}+U\left(\left|x_{2}\right|\right)
$$

so that

$$
H_{T}\left(x_{2}\right) \phi_{n}\left(x_{2}\right)=\epsilon_{n} \phi_{n}\left(x_{2}\right)
$$

Again, we restrict ourselves to the even case $\phi_{n}\left(x_{2}\right)=\phi_{n}\left(-x_{2}\right)$. If we choose a box of width $2 L$ with infinite walls as confining potential the transverse eigenfunction system is given by (3.6). Projection of (3.9) on the transverse modes gives 


$$
\begin{aligned}
& H_{n m}=\left\langle\phi_{n}|H| \phi_{m}\right\rangle=\left[-\frac{1}{2} \partial_{x_{1}}^{2}+\epsilon_{n}\right] \delta_{n m}+\hat{V}_{n m}\left(x_{1}\right) \\
& \hat{V}_{n m}=\int_{-L}^{L} d x_{2} \phi_{n}\left(x_{2}\right) \phi_{m}\left(x_{2}\right) V\left(x_{1}, x_{2}\right) .
\end{aligned}
$$

A matrix of longitudinal momenta can be defined as in (3.8). The reference momentum $k_{F}$ of the two electron problem can now be interpreted as the one electron Fermi momentum and the open channels are characterized by the condition $k_{n}=\sqrt{2 \epsilon_{n}}<k_{F}$. Comparing (3.7) with (3.13) we see that both the strip problem and the two electron problem are described by very similar quasi 1d (many channel) Hamiltonians. The only important difference resides in the definition of $\hat{V}_{n m}(x)$ in the two cases. In the next step we apply the transfer matrix method to (3.7) and (3.13).

We define a longitudinal $N$-channel wavefunction $\psi(x)^{T}=\left(\psi_{1}(x), \ldots, \psi_{N}(x)\right)$ so that the Schrödinger equation for a Hamiltonian of the type (3.7) or (3.13) reads

$$
E \psi(x)=H \psi(x)=-\frac{1}{2} \psi^{\prime \prime}(x)+\hat{\epsilon} \psi(x)+\hat{V} \psi(x)
$$

where $\hat{\epsilon}$ is a diagonal matrix with $(\hat{\epsilon})_{n n}=\epsilon_{n}$ and the primes in $\psi^{\prime \prime}(x)$ indicate the derivative with respect to $x$. We introduce a transfer matrix $A$ by

$$
\left(\begin{array}{c}
\psi(x) \\
\psi^{\prime}(x)
\end{array}\right)^{\prime}=\left(\begin{array}{cc}
0 & 1 \\
2(\hat{\epsilon}-E+\hat{V}) & 0
\end{array}\right)\left(\begin{array}{c}
\psi(x) \\
\psi^{\prime}(x)
\end{array}\right) \equiv A\left(\begin{array}{c}
\psi(x) \\
\psi^{\prime}(x)
\end{array}\right)
$$

Redefining the wavefunction through

$$
\tilde{v}(x)=\frac{1}{\sqrt{2 \kappa}}\left(\begin{array}{c}
\psi^{\prime}(x)+i \kappa \psi(x) \\
\psi^{\prime}(x)-i \kappa \psi(x)
\end{array}\right) \equiv C\left(\begin{array}{c}
\psi(x) \\
\psi^{\prime}(x)
\end{array}\right)
$$

(we recall that $\kappa$ is the diagonal matrix (3.8)) we have $\tilde{v}^{\prime}(x)=C A C^{-1} \tilde{v}(x) \equiv \tilde{T}(x) \tilde{v}(x)$ with

$$
\tilde{T}(x)=i\left(\begin{array}{cc}
\kappa & 0 \\
0 & -\kappa
\end{array}\right)-\frac{i}{\sqrt{\kappa}}\left(\begin{array}{cc}
\hat{V}(x) & -\hat{V}(x) \\
\hat{V}(x) & -\hat{V}(x)
\end{array}\right) \frac{1}{\sqrt{\kappa}} .
$$

To get rid of the first matrix on the r.h.s. of (3.17) we redefine the wavefunction again,

$$
\tilde{v}(x)=\left(\begin{array}{cc}
e^{i \kappa x} & 0 \\
0 & e^{-i \kappa x}
\end{array}\right) v(x)
$$

and finally arrive at

$$
v^{\prime}(x)=T(x) v(x)
$$

with

$$
T(x)=-\frac{i}{\sqrt{\kappa}}\left(\begin{array}{cc}
e^{-i \kappa x} \hat{V}(x) e^{i \kappa x} & -e^{-i \kappa x} \hat{V}(x) e^{-i \kappa x} \\
e^{i \kappa x} \hat{V}(x) e^{i \kappa x} & -e^{i \kappa x} \hat{V}(x) e^{-i \kappa x}
\end{array}\right) \frac{1}{\sqrt{\kappa}} .
$$

It is not difficult to see that (3.19) is equivalent to the following evolution law for the full transfer matrix $\hat{M}(x)$, 


$$
\hat{M}(x+\delta x)=e^{\delta \hat{M}} \hat{M}(x)
$$

where the "building block" $\delta \hat{M}$ is given by

$$
\delta \hat{M}=\int_{x}^{x+\delta x} T\left(x^{\prime}\right) d x^{\prime}=-\frac{i}{\sqrt{\kappa}}\left(\begin{array}{ll}
\delta v_{1} & -\delta v_{2} \\
\delta v_{2}^{\dagger} & -\delta v_{1}^{\dagger}
\end{array}\right) \frac{1}{\sqrt{\kappa}} \equiv\left(\begin{array}{cc}
a & b \\
b^{\dagger} & d
\end{array}\right)
$$

with

$$
\begin{aligned}
& \delta v_{1}=\int_{x}^{x+\delta x} d x^{\prime} e^{-i \kappa x^{\prime}} \hat{V}\left(x^{\prime}\right) e^{i \kappa x^{\prime}} \\
& \delta v_{2}=\int_{x}^{x+\delta x} d x^{\prime} e^{-i \kappa x^{\prime}} \hat{V}\left(x^{\prime}\right) e^{-i \kappa x^{\prime}}
\end{aligned}
$$

Under the crucial assumptions that both the blocks of $T(x)$ in $(3.20)$ and all the matrix elements within these blocks are statistically independent, we deal with the so-called isotropic situation and the evolution law (3.21) leads to the Dorokhov-Mello-Pereyra-Kumar (DMPK) equation [11] for the eigenvalue distribution of $\hat{M}(x)$. This equation accurately describes the transport of independent electrons in quasi $1 \mathrm{~d}$ wires and is - at least in the unitary case - completely under control [12, 13]. In the case of a $2 \mathrm{~d}$ strip of width not significantly exceeding the elastic mean free path $l_{e l}$ the exponential factors $e^{ \pm i \kappa x}$ in (3.20) oscillate rapidly so that the assumption of statistically independent matrix elements seems very reasonable. This argument provides a microscopic justification for the application of the DMPK equation to quasi 1d wires [11]. A main purpose of our present study is to point out that the DMPK equation also describes the transport of two interacting electrons in a disordered 1d system, provided the pair size does not exceed $l_{e l}$. This statement should be practically obvious since both the strip and the two electron problem have been formulated in almost identical technical terms: The DMPK equation is as well-justified for the latter problem as for the former.

\section{B. TIP-Localization Length}

Once the applicability of DMPK theory is guaranteed the next step is to identify the localization length of the problem in terms of the given system parameters. The connection between the building block (3.22) and the localization length $\xi$ in the DMPK approach is given by [11]

$$
\frac{N^{2}}{\xi} \delta x=\left\langle\operatorname{tr}\left(b^{\dagger} b\right)\right\rangle
$$

i.e.

$$
\frac{N^{2}}{\xi} \delta x=\int_{x}^{x+\delta x} d x_{1} d x_{2}\left\langle\operatorname{tr}\left[\frac{1}{\kappa} e^{-i \kappa\left(x_{1}-x_{2}\right)} \hat{V}\left(x_{1}\right) \frac{1}{\kappa} e^{-i \kappa\left(x_{1}-x_{2}\right)} \hat{V}\left(x_{2}\right)\right]\right\rangle
$$

Any further evaluation of (3.25) depends on the precise form of $\hat{V}(x)$ and there, finally, the differences between the two cases studied here come in. 
Let us begin with the disordered strip. Inserting $\hat{V}(x)$ as defined in $(3.13)$ in (3.25) we get

$$
\begin{gathered}
\frac{N^{2}}{\xi} \delta x=\int_{x}^{x+\delta x} d x_{1} d x_{2} \int_{-L}^{L} d y_{1} d y_{2} t r\left[\frac{1}{\kappa} e^{-i \kappa\left(x_{1}-x_{2}\right)} \phi\left(y_{1}\right) \phi\left(y_{1}\right)^{T} \frac{1}{\kappa} e^{-i \kappa\left(x_{1}-x_{2}\right)} \phi\left(y_{2}\right) \phi\left(y_{2}\right)^{T}\right] \\
\left\langle V\left(x_{1}, y_{1}\right) V\left(x_{2}, y_{2}\right)\right\rangle
\end{gathered}
$$

and after using (3.10) and then (3.6)

$$
\begin{aligned}
\frac{N^{2}}{\xi} \delta x & =c_{2} \delta x \int_{-L}^{L} d y \sum_{n m} \frac{1}{\kappa_{n} \kappa_{m}} \phi_{n}(y)^{2} \phi_{m}(y)^{2} \\
& =\frac{c_{2}}{2 L} \delta x \sum_{n m} \frac{1}{\kappa_{n} \kappa_{m}}\left(1+\frac{1}{2} \delta_{n m}\right) .
\end{aligned}
$$

Evaluating the remaining sums approximately (see appendix B),

$$
\begin{aligned}
& \sum_{n=0}^{N-1} \frac{1}{\kappa_{n}} \approx \frac{L}{2}, \\
& \sum_{n=0}^{N-1} \frac{1}{\kappa_{n}^{2}} \approx \frac{L^{2}}{2 \pi^{2}} \frac{\ln 2 N}{N}
\end{aligned}
$$

we can neglect the diagonal contribution in (3.27) as a $1 / N$ correction. Together with (see appendix Q)

$$
c_{d} \approx \frac{\pi^{d-1}}{d} \frac{k_{F}^{4-d}}{k_{F} l_{e l}}
$$

for $d=2$ we finally arrive at

$$
\frac{N^{2}}{\xi}=\frac{\pi}{16} \frac{k_{F} L}{l_{e l}} \Leftrightarrow \xi=\frac{16}{\pi^{2}} N l_{e l}
$$

This is the well-known relation between the localization length and the elastic mean free path in quasi $1 \mathrm{~d}$ disordered strips. In the following we will show that a similar relation also holds in the case of two electrons with a bag interaction, although the detailed reasoning is a little more involved than in the previous case. From (3.7) and (3.25) we have (instead of (3.26))

$$
\begin{gathered}
\frac{N^{2}}{\xi} \delta x=4 \int_{x}^{x+\delta x} d x_{1} d x_{2} \int_{-L}^{L} d y_{1} d y_{2} \operatorname{tr}\left[\frac{1}{\kappa} e^{-i \kappa\left(x_{1}-x_{2}\right)} \phi\left(y_{1}\right) \phi\left(y_{1}\right)^{T} \frac{1}{\kappa} e^{-i \kappa\left(x_{1}-x_{2}\right)} \phi\left(y_{2}\right) \phi\left(y_{2}\right)^{T}\right] \\
\left\langle\left[V\left(x_{1}+y_{1} / 2\right)+V\left(x_{1}-y_{1} / 2\right)\right]\left[V\left(x_{2}+y_{2} / 2\right)+V\left(x_{2}-y_{2} / 2\right)\right]\right\rangle
\end{gathered}
$$

According to $(3.2)$ the average over the random potential leads to four $\delta$-functions so that $x_{1}-x_{2}=\left(s_{1} y_{1}+s_{2} y_{2}\right) / 2$ with $s_{1,2}= \pm 1$ (independently). Since the typical scale for $\delta x$ is $l_{e l}$ the $\delta$-function condition can be always fulfilled and we arrive at

$$
\begin{aligned}
\frac{N^{2}}{\xi} \delta x=4 c_{1} \delta x \sum_{s_{1}, s_{2}= \pm 1} \operatorname{tr} & {\left[\left(\frac{1}{\kappa} \int d y_{1} e^{-i \kappa s_{1} y_{1} / 2} \phi\left(y_{1}\right) \phi\left(y_{1}\right)^{T} e^{-i \kappa s_{1} y_{1} / 2}\right)\right.} \\
& \left.\left(\frac{1}{\kappa} \int d y_{2} e^{-i \kappa s_{2} y_{2} / 2} \phi\left(y_{2}\right) \phi\left(y_{2}\right)^{T} e^{-i \kappa s_{2} y_{2} / 2}\right)\right]
\end{aligned}
$$


or

$$
\frac{N^{2}}{\xi}=16 c_{1} \operatorname{tr}\left[\frac{1}{\kappa} B \frac{1}{\kappa} B\right],
$$

where

$$
B_{n m}=\int_{-L}^{L} d y \cos \left(\left(\kappa_{n}+\kappa_{m}\right) y / 2\right) \phi_{n}(y) \phi_{m}(y)
$$

Employing (3.29) for $d=1$ and introducing $\eta_{n}=\sqrt{N^{2}-(n+1 / 2)^{2}}$, (3.33) can be rewritten as

$$
\frac{1}{\xi}=\frac{4}{l_{e l}} \sum_{n m} \frac{1}{\eta_{n} \eta_{m}} B_{n m}^{2}
$$

In appendix $\mathrm{D}$ it is shown that (3.35) leads to the final result

$$
\xi \approx \frac{N l_{e l}}{2 \sqrt{2} \ln (1+\sqrt{2})} .
$$

Using $N=k_{F} L / \pi$ and $L \approx l_{e l}$ (which is the maximal pair size allowed within the present technical framework) we have

$$
\xi \sim k_{F} l_{e l}^{2}
$$

a result which has first been derived by Dorokhov [24] for two particles interacting via a harmonic potential. Eq. (3.37) shows that the localization length for two interacting particles is significantly enhanced over the one-particle value $\xi \sim l_{e l}$.

\section{Scaling of the TIP-Conductance}

In conclusion, we have demonstrated the validity of the DMPK equation for the transport properties of two electrons in a narrow bag in 1d. As our main argument we have pointed out the almost complete analogy to the the case of a disordered quasi $1 \mathrm{~d}$ strip. Identifying the localization length in terms of the system parameters we recovered Dorokhov's result (3.37).

As probably the most important consequence of our investigation we can identify, as in the $\sigma$ model formulation above, a scaling parameter in the two electron problem, namely the conductance $g=2 \operatorname{tr}\left[\left(\hat{M}^{\dagger} \hat{M}+\left(\hat{M}^{\dagger} \hat{M}\right)^{-1}+2\right)^{-1}\right]$ of the associated strip problem. Although this quantity does not seem to have an immediate physical interpretation for two interacting electrons and should perhaps be regarded as some sort of auxiliary variable it is nevertheless of considerable value: Its length dependence in the whole range from the diffusive to the localized regime is under control [10] and the crossover point between these regimes is given by $g(\xi)=1$. In this way we have again justified the assumptions that underly Imry's application [17] of the Thouless block picture to the problem of interacting particles in a random potential. We think that Imry's pair conductance $g_{2}$ should be identified with our conductance $g$ of the associated strip problem. This identification explains the remarkable success of the scaling block picture in the present context and clarifies the nature of the scaling parameter. 


\section{Generalization to Many Particles}

The above considerations for two interacting particles in a random potential can be generalized to larger particle numbers provided the particle density (in the case of Fermions) is low enough to neglect effects of the Pauli principle. In this paper we refrain from discussing the full many body problem, where quasiparticles are the relevant degrees of freedom. A particularly important issue in this context would be the lifetime of interacting quasiparticles as a function of their excitation energy. For a first numerical study of the influence of the Pauli principle on interaction-assisted coherent transport see [34].

Let us consider the $M$-particle Hamiltonian

$$
H=-\frac{1}{2} \sum_{i=1}^{M}\left(\partial_{x_{i}}^{2}+V\left(x_{i}\right)\right)+\sum_{i<j} U\left(\left|x_{i}-x_{j}\right|\right)
$$

where $U\left(\left|x-x^{\prime}\right|\right)$, as before, represents an attractive bag of size $2 L$. Introducing the center of mass coordinate $x$ and $M-1$ relative coordinates,

$$
\begin{aligned}
x & =\frac{1}{M} \sum_{i=1}^{M} x_{i}, \quad(j=1, \ldots, M-1), \\
y_{j} & =x_{j}-x_{j+1} \quad,
\end{aligned}
$$

we get

$$
\begin{aligned}
H= & -\frac{1}{2 M} \partial_{x}^{2}-f\left(\partial_{y_{1}}, \ldots, \partial_{y_{M-1}}\right)+\sum_{i=1}^{M} V\left(x+g_{i}\left(y_{1}, \ldots, y_{M-1}\right)\right) \\
& +\sum_{i<j} U\left(h_{i j}\left(y_{1}, \ldots, y_{M-1}\right)\right)
\end{aligned}
$$

Here, $f, g_{i}$, and $h_{i j}$ are functions which we do not have to specify in detail for our subsequent considerations. In complete analogy to the previous section we can define a transverse Hamiltonian and its eigenfunction system by

$$
\begin{aligned}
& H_{T}=-f\left(\partial_{y_{1}}, \ldots, \partial_{y_{M-1}}\right)+\sum_{i<j} U\left(h_{i j}\left(y_{1}, \ldots, y_{M-1}\right)\right) \\
& H_{T} \varphi_{n_{1} \ldots n_{M-1}}\left(y_{1}, \ldots, y_{M-1}\right)=\epsilon_{n_{1} \ldots n_{M-1}} \varphi_{n_{1} \ldots n_{M-1}}\left(y_{1}, \ldots, y_{M-1}\right)
\end{aligned}
$$

We do not specify any particular symmetry for the transverse eigenfunctions $\varphi_{n_{1} \ldots n_{M-1}}\left(y_{1}, \ldots, y_{M-1}\right)$. Projecting the full Hamiltonian $H$ on the transverse eigenfunction system we arrive at

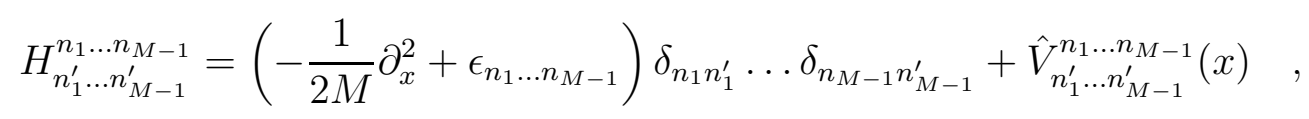

where

$$
\begin{aligned}
& \hat{V}_{n_{1}^{\prime} \ldots n_{M-1}^{\prime}}^{n_{1} \ldots n_{M-1}}(x)=\int d y_{1} \ldots d y_{M-1} \varphi_{n_{1} \ldots n_{M-1}}\left(y_{1}, \ldots, y_{M-1}\right) \\
& \left(\sum_{i=1}^{M} V\left(x+g_{i}\left(y_{1}, \ldots, y_{M-1}\right)\right)\right) \varphi_{n_{1}^{\prime} \ldots n_{M-1}^{\prime}}\left(y_{1}, \ldots, y_{M-1}\right)
\end{aligned}
$$


Defining the "building block" $\delta M$ as in (3.22) and keeping in mind the relation (3.24) we have

$$
\left\langle\operatorname{tr}\left(b^{\dagger} b\right)\right\rangle=\int_{x}^{x+\delta x} d x_{1} d x_{2}\left\langle\operatorname{tr}\left(\frac{1}{\kappa} e^{-i \kappa\left(x_{1}-x_{2}\right)} \hat{V}\left(x_{1}\right) \frac{1}{\kappa} e^{-i \kappa\left(x_{1}-x_{2}\right)} \hat{V}\left(x_{2}\right)\right)\right\rangle,
$$

where $\kappa_{n_{1} \ldots n_{M-1}}=\sqrt{2\left(E-\epsilon_{n_{1} \ldots n_{M-1}}\right)}$. The relevant average in (3.44) is straightforwardly calculated,

$$
\begin{aligned}
&\left\langle\sum _ { i = 1 } ^ { M } V \left( x_{1}+\right.\right.\left.\left.g_{i}\left(y_{1} \ldots y_{M-1}\right)\right) \sum_{j=1}^{M} V\left(x_{2}+g_{j}\left(y_{1}^{\prime} \ldots y_{M-1}^{\prime}\right)\right)\right\rangle= \\
& \frac{k_{F}^{3}}{2 k_{f} l_{e l}} \sum_{i, j=1}^{M} \delta\left(x_{1}-x_{2}+g_{i}\left(y_{1} \ldots y_{M-1}\right)-g_{j}\left(y_{1}^{\prime} \ldots y_{M-1}^{\prime}\right)\right)
\end{aligned} .
$$

Both the $x_{1}$ and the $x_{2}$ integration in (3.44) can be easily performed and we get

$$
\begin{aligned}
&\left\langle\operatorname{tr}\left(b^{\dagger} b\right)\right\rangle= \frac{k_{F}^{3}}{2 k_{F} l_{e l}} \delta x \sum_{i, j=1}^{M} \int d y_{1} \ldots d y_{M-1} d y_{1}^{\prime} \ldots d y_{M-1}^{\prime} \\
& \operatorname{tr}\left(\frac{1}{\kappa} e^{-i \kappa\left(g_{j}-g_{i}\right)} \varphi\left(y_{1}, \ldots, y_{M-1}\right) \varphi\left(y_{1}, \ldots, y_{M-1}\right)^{T}\right. \\
&\left.\quad \frac{1}{\kappa} e^{-i \kappa\left(g_{j}-g_{i}\right)} \varphi\left(y_{1}^{\prime}, \ldots, y_{M-1}^{\prime}\right) \varphi\left(y_{1}^{\prime}, \ldots, y_{M-1}^{\prime}\right)^{T}\right) \\
&=\frac{k_{F}^{3}}{2 k_{F} l_{e l}} \delta x \operatorname{tr}\left(\frac{1}{\kappa} B \frac{1}{\kappa} B^{\dagger}\right)
\end{aligned}
$$

with

$$
\begin{gathered}
B_{n_{1}^{\prime} \ldots n_{M-1}^{\prime}}^{n_{1} \ldots n_{M-1}}=\int_{-L}^{L} d y_{1} \ldots d y_{M-1}\left(\sum_{i=1}^{M} e^{i\left(\kappa_{n_{1} \ldots n_{M-1}}+\kappa_{n_{1}^{\prime} \ldots n_{M-1}^{\prime}}\right) g_{i}\left(y_{1}, \ldots, y_{M-1}\right)}\right) \\
\varphi_{n_{1} \ldots n_{M-1}}\left(y_{1}, \ldots, y_{M-1}\right) \varphi_{n_{1}^{\prime} \ldots n_{M-1}^{\prime}}\left(y_{1}, \ldots, y_{M-1}\right)
\end{gathered}
$$

which corresponds to (3.34). For further progress we have to rely on certain estimates. Let us assume that the phase factor $\exp \left(i\left(\kappa_{n_{1} \ldots n_{M-1}}+\kappa_{n_{1}^{\prime} \ldots n_{M-1}^{\prime}}\right) g_{i}\left(y_{1}, \ldots, y_{M-1}\right)\right)$ fluctuates strongly when the $y_{i}$ vary between $-L$ and $L$. Then it can be replaced by its average denoted by $c_{p h}$ and we have

$$
B_{n_{1}^{\prime} \ldots n_{M-1}^{\prime}}^{n_{1} \ldots n_{M-1}} \approx c_{p h} \delta_{n_{1} \ldots n_{1}^{\prime}} \ldots \delta_{n_{M-1} n_{M-1}^{\prime}}
$$

Our assumption is only correct if $\kappa_{n_{1} \ldots n_{M-1}} \gg 1 / L$. This means that channels with high transverse excitation energies and small longitudinal momenta $\kappa_{n_{1} \ldots n_{M-1}}$ are not correctly described by our approximation. Comparing the result of our estimate with the more accurate calculation for $M=2$ in the previous section we can determine the error incurred. Using (3.24) and (3.48) we have

$$
\frac{1}{\xi}=\frac{c_{p h}^{2}}{N^{2}} \frac{k_{F}^{3}}{2 k_{F} l_{e l}} \sum_{n_{1} \ldots n_{M-1}} \frac{1}{\kappa_{n_{1} \ldots n_{M-1}}^{2}} .
$$


We estimate the sum in (3.49) by an integral approximation,

$$
\sum_{n_{1} \ldots n_{M-1}} \frac{1}{\kappa_{n_{1} \ldots n_{M-1}}^{2}} \approx \int_{0}^{E} d \epsilon \rho(\epsilon) \frac{1}{2(E-\epsilon)}
$$

where $E$ is the $M$-particle energy at which we study the transport problem, and $\rho(\epsilon)$ is the density of transverse energy levels. To continue, we replace $\rho(\epsilon)$ by its average $\bar{\rho}$ and assume that $E-\epsilon \approx \Delta$ (with $\Delta$ the transverse level spacing) provides the cutoff for the otherwise divergent integral in (3.50):

$$
\begin{aligned}
\int_{0}^{E} d \epsilon \rho(\epsilon) \frac{1}{2(E-\epsilon)} & =\frac{1}{2} \int_{0}^{1} d x \frac{\rho(x)}{1-x} \\
& \approx \frac{\bar{\rho}}{2} \int_{0}^{1-\delta} \frac{d x}{1-x} \\
& =-\frac{\bar{\rho}}{2} \ln \delta .
\end{aligned}
$$

With $E \sim k_{F}^{2}$, the number of transverse channels $N=\left(k_{F} L\right)^{M-1}, \bar{\rho}=N / E$, and $\delta=\Delta / E=$ $1 / N$ we finally arrive at (disregarding numerical prefactors)

$$
\xi \sim \frac{1}{c_{p h}^{2}} \frac{\left(k_{F} L\right)^{M-1} l_{e l}}{\ln \left[\left(k_{F} L\right)^{M-1}\right]} .
$$

For the case $M=2$ we almost recover the result (3.37) of the previous section. The logarithmic suppression in (3.52) is an artifact of our approximations. It arises precisely from the levels with $E \approx \epsilon$, i.e. $\kappa \approx 0$, which are not well-described by our approach. Therefore we discard the $\ln$-correction in (3.52) and have as our final result (for $L \approx l_{e l}$ )

$$
\xi \sim\left(k_{F} l_{e l}\right)^{M-1} l_{e l}
$$

This shows how the enhancement factor for coherent multiple particle propagation increases with particle number $M$.

\section{CONCLUSION AND DISCUSSION}

In this work, we have developed and further improved two analytical approaches to the phenomenon of interaction-assisted coherent pair propagation. One, the $\sigma$ model formulation based on an effective random Hamiltonian, was inspired by Shepelyansky's view 16. The other, a transfer matrix approach to two or many tightly bound particles, was built on the pioneering ideas of Dorokhov 24]. In its most succinct form, the message from our investigations is the following: Transport of interacting pairs or of small aggregates of particles can be described in terms which have been introduced in the context of, and are therefore familiar from, one-electron theory.

In particular, pair transport is governed by a scaling parameter, the effective two-particle conductivity. This is a rigorous statement, once the models introduced above are accepted, and adds to our confidence in Imry's generalization [17] of the Thouless scaling picture. 
Furthermore, there is an energy scale, the two-particle Thouless energy, associated with diffusive pair transport. It corresponds, as in one-electron theory, to the time it takes to diffuse through the sample and defines the scale, for which deviations from random matrix theory occur in the spectral statistic of diffusing pair states. The precise pair dynamics, however, differs from the one-particle case in that both the diffusion constant and the density of states are frequency dependent. This is a clear manifestation of the pairs' composite character, since it is connected with a logarithmic growth of the pair size and a corresponding logarithmic attenuation of the diffusion constant as a function of time. Apart from confirming important aspects of these conclusions, our second (transfer matrix) approach enabled us to study, in a rather general form, the behavior of more than two tightly bound particles. It turned out that the enhancement factor, i.e. the ratio between the $M$-particle and the one-particle localization length, grows as the $(M-1)$ th power of the latter.

Of course, both models introduced in this paper have their shortcomings. Foremost, our $\sigma$ model formulation relies heavily on the assumption that the effective Hamiltonian catches the essential physics of the problem. We can imagine two types of criticism: (i) quantitative statistical assumptions like the size of the fluctuating interaction matrix elements or the form of the decay towards the band edges are incorrect or unrealistic. Corresponding modifications would lead to quantitative corrections without precluding our whole approach. (ii) Qualitative assumptions like the statistical independence of the matrix elements are incorrect in an essential way. This would call into question the $\sigma$ model formulation as such, since it is hard to imagine how a similar $\sigma$ model can be obtained in the presence of important correlations. One should also note that the one-particle localization length $L_{1}$ is the smallest length scale resolved by the effective Hamiltonian. For length scales $L<L_{1}$ effects associated with the transport of single particles have to be taken into account [35]. In this case the interpretation of the two-particle conductance is less straightforward.

The major drawback of the transfer matrix model, on the other hand, is its restriction to tightly bound particles. This restriction is necessary to prevent the disorder from being relevant for the relative motion of the particles. Otherwise relative and center of mass motion would have been coupled and the problem much harder to solve. Naturally, however, the requirement of strong binding restricts the physical situations to which the model may be applied.

Combined, the two approaches explained in this paper represent our analytical knowledge concerning interaction-assissted coherent transport. For the future, we believe that it is an important and worthwhile task to devise and investigate an effective Hamiltonian for more than two particles. This would be another step towards understanding many-particle effects and it would be free of the constraints inevitably associated with the transfer matrix method. It is also of interest to investigate the question, whether the effect discussed here is related to a mechanism for the enhancement of persistent currents proposed some time ago [36].

\section{ACKNOWLEDGMENT}

We are grateful to Y. Imry, D.L. Shepelyansky and D. Weinmann for fruitful discussions and their interest in our work. AMG would also like to acknowledge discussions with S. Grossmann, D. Lohse, A.D. Mirlin, F. von Oppen, R. Römer, M. Schreiber, and P. Wölfle. 
This work was supported by the European HCM program (KF) and a NATO fellowship through the DAAD (AMG).

\section{APPENDIX A: EXPANSION OF THE EFFECTIVE POTENTIAL (2.32)}

In this Appendix, we derive an expression for the effective $Q$-potential $f_{R}(Q)$ which is more suitable for the comparison with the standard $\sigma$ model of a disordered metal $\mathcal{L}_{\text {met }}[Q]$. For this, we consider a quantity like

$$
I=\operatorname{str} \ln (a+b \Lambda+c Q)
$$

with some complex parameters $a, b, c$ and $\Lambda, Q$ as in (2.32). In the grading for advanced and retarded Green's functions, $\Lambda$ takes the form $\Lambda=\left(\begin{array}{cc}1 & 0 \\ 0 & -1\end{array}\right)$. The quantity $I$ only depends on the radial parameters $\theta$ [5] of $Q=T^{-1} \Lambda T$, i.e. we may choose $T=\exp \left(\theta \sigma_{1}\right)$, where $\sigma_{1}=\left(\begin{array}{ll}0 & 1 \\ 1 & 0\end{array}\right)$ and $\theta$ is $4 \times 4$ supermatrix with two or three independent radial parameters [5] (depending on the symmetry class). Then we have $\sigma_{1} \Lambda \sigma_{1}=-\Lambda$ and $\sigma_{1} Q \sigma_{1}=-Q$. Inserting $1=\sigma_{1}^{2}$ and permuting the matrix products in the argument of the logarithm in (A1), we thus obtain:

$$
\begin{aligned}
I & =\operatorname{str} \ln (a-b \Lambda-c Q)=\frac{1}{2} \operatorname{str} \ln ((a+b \Lambda+c Q)(a-b \Lambda-c Q))= \\
& =\frac{1}{2} \operatorname{str} \ln \left(\left[a^{2}-(b+c)^{2}\right]-b c[Q \Lambda+\Lambda Q-2]\right) .
\end{aligned}
$$

We now expand $I$, for the case in which either $|b c| \ll\left|a^{2}-(b+c)^{2}\right|$ is valid or the matrix $\Delta Q=(Q \Lambda+\Lambda Q-2)$ may be considered as small. The latter condition is clearly valid for a perturbative treatment where $Q$ can be parameterized [5] as $Q=\Lambda \frac{1+i P}{1-i P} \simeq \Lambda(1+2 i P+$ $\left.(2 i P)^{2}+\cdots\right)$ with $\Lambda P \Lambda=-P$. Then $\Delta Q=8(i P)^{2}+\mathcal{O}\left(P^{4}\right)$ is indeed a small parameter and expanding ( $\mathrm{A2})$, we find:

$$
I \simeq-\frac{b c}{a^{2}-(b+c)^{2}} \operatorname{str}(Q \Lambda)
$$

Applying (A3) on $f_{R}(Q)$ defined in (2.32), we obtain:

$$
f_{R}(Q) \simeq-\frac{1}{2}\left(\sum_{j} \frac{\left(\frac{1}{2} \omega\right)\left(\frac{i}{2} \Gamma_{1} v(j)\right)}{\left(E-\eta_{j}^{R}+\frac{1}{2} \Gamma_{0} v(j)\right)^{2}-\left(\frac{1}{2} \omega+\frac{i}{2} \Gamma_{1} v(j)\right)^{2}}\right) \operatorname{str}(Q \Lambda)
$$

Here, the terms with $|j| \lesssim L_{c} \sim L_{1} \ln L_{\text {sys }}$ can be considered as selfaveraging with respect to the random variables $\eta_{j}^{R}$, due to overlapping resonances. The typical (most probable) values of the remaing terms (with $|j| \gtrsim L_{c}$ ) are exponentially small since $v(j) \sim e^{-4|j| / L_{1}}$. They can be neglected whereas the other terms may be replaced by their $\eta$-average. In addition, we assume that the integration range $2 W_{b}$ for $\eta$ is much larger than the other energy scales $\omega, \Gamma_{0}, \Gamma_{1}$ and that the $\eta$-density $\rho_{0}(\eta)$ varies rather slowly on these "small" scales. The $\eta$-integral may then be extended to all $\eta$ with $-\infty<\eta<\infty$ and $\rho_{0}(\eta)$ can be replaced by $\rho_{0}(E)$. Performing the $\eta$-integration, we find: 


$$
f_{R}(Q) \simeq-i \frac{\pi}{4} \omega h\left(\frac{\Gamma_{1}}{\omega}\right) \rho_{0}(E) \operatorname{str}(Q \Lambda)
$$

with

$$
h\left(\frac{\Gamma_{1}}{\omega}\right)=i \frac{\Gamma_{1}}{\omega} \sum_{|j| \lesssim L_{c}} \frac{v(j)}{1+i \frac{\Gamma_{1}}{\omega} v(j)} .
$$

In order to obtain a more explicit expression for this function, we would have to specify the choice for $v(j)$. However, the relevant limiting cases are:

$$
\begin{aligned}
& h\left(\frac{\Gamma_{1}}{\omega}\right) \simeq i \frac{\Gamma_{1}}{\omega}\left(S_{1}-i \frac{\Gamma_{1}}{\omega} S_{2}+\cdots\right) \quad, \quad S_{\nu}=\sum_{j}[v(j)]^{\nu} \quad, \quad \Gamma_{1} \ll|\omega|, \\
& h\left(\frac{\Gamma_{1}}{\omega}\right) \simeq\left[\frac{L_{1}}{4} \ln \left(\frac{\Gamma_{1}}{|\omega|}\right)\right]^{d}, \quad \tau_{c}^{-1} \lesssim|\omega| \ll \Gamma_{1}, \\
& h\left(\frac{\Gamma_{1}}{\omega}\right) \simeq\left[\frac{L_{1}}{4} \ln \left(\Gamma_{1} \tau_{c}\right)\right]^{d}=L_{c}^{d} \quad, \quad|\omega| \ll \tau_{c}^{-1} .
\end{aligned}
$$

Here, $\tau_{c}^{-1}=\Delta_{\text {eff }}=\Gamma_{1} v\left(L_{c}\right)$ is the effective level spacing of the well coupled pair states, introduced subsequent to Eq. (2.28). The cutoff in the sum (A6) is either given by $|j| \lesssim$ $L_{\text {eff }}(\omega)\left[\right.$ cp. (2.29)] or $|j| \lesssim L_{c}$, resulting in (A8) and (A9), respectively.

\section{APPENDIX B: SOME ESTIMATES}

In this appendix we estimate the discrete sums $\sum_{n}\left(1 / \kappa_{n}\right), \sum_{n}\left(1 / \kappa_{n}^{2}\right)$ contributing in (3.27). With $2 E \approx N^{2} \pi^{2} / L^{2}, k_{n}^{2}=\pi^{2}(n+1 / 2)^{2} / L^{2} \approx n^{2} \pi^{2} / L^{2}$, and approximating the sum by an integral we have

$$
\begin{aligned}
\sum_{n=0}^{N-1} \frac{1}{\kappa_{n}} & \approx \int_{0}^{N} \frac{d n}{\sqrt{2 E-k_{n}^{2}}} \\
& \approx \frac{L}{\pi N} \int_{0}^{N} \frac{d n}{\sqrt{1-n^{2} / N^{2}}} \\
& =\frac{L}{\pi} \int_{0}^{1} \frac{d x}{\sqrt{1-x^{2}}} \\
& =L / 2 .
\end{aligned}
$$

Analogously, we get

$$
\sum_{n=0}^{N-1} \frac{1}{\kappa_{n}^{2}} \approx \frac{L^{2}}{\pi^{2} N} \int_{0}^{1} \frac{d x}{1-x^{2}}
$$

which is logarithmically divergent. However, we have to take into account the energy difference between $E$ and the transverse energy of the last channel. This energy gap is of the order of the level spacing and hence of relative magnitude $1 / N$. Therefore, 


$$
\begin{aligned}
\sum_{n=0}^{N-1} \frac{1}{\kappa_{n}^{2}} & \approx \frac{L^{2}}{\pi^{2} N} \int_{0}^{1-1 / N} \frac{d x}{1-x^{2}} \\
& =\left.\frac{L^{2}}{\pi^{2} N} \frac{1}{2} \ln \frac{1+x}{1-x}\right|_{0} ^{1-1 / N} \\
& \approx \frac{L^{2}}{2 \pi^{2}} \frac{\ln 2 N}{N}
\end{aligned}
$$

This completes the derivation of (3.28).

\section{APPENDIX C: NORMALIZATION OF THE RANDOM POTENTIAL}

To estimate the constants $c_{1}$ and $c_{2}$ appearing in (3.2) and (3.10), respectively, we recall the normalization condition for models with random white noise disorder [5],

$$
\left\langle V(\vec{x}) V\left(\vec{x}^{\prime}\right)=\frac{1}{2 \pi \nu \tau} \delta\left(\vec{x}-\vec{x}^{\prime}\right)\right.
$$

where $\nu$ is the local density of states and $\tau$ the elastic mean free time (we put $\hbar=m=1$ ).

With $\tau=l_{e l} / k_{F}$ and $N=\left(k_{F} L / \pi\right)^{d}$ in $d$ dimensions we get for the total density of states

$$
\rho=\frac{d N}{d E}=\frac{d}{2} k_{f}^{d-2}\left(\frac{L}{\pi}\right)^{d}
$$

and hence

$$
\nu=\frac{\rho}{L^{d}}=\frac{d}{2} k_{F}^{d-2} \pi^{-d}
$$

so that

$$
c_{d}=\frac{1}{2 \pi \nu \tau}=\frac{\pi^{d-1}}{d} \frac{k_{f}^{4-d}}{k_{F} l_{e l}}
$$

which is exactly 3.29$)$.

\section{APPENDIX D: FINAL EVALUATION OF $1 / \xi$}

We have to estimate the r.h.s. of (3.35). Using (3.6) and (3.34) an elementary calculation shows that

$$
B_{n m}=\frac{1}{2} \sum_{s_{1}, s_{2}= \pm 1} \frac{\sin \left[\left(\kappa_{n}+\kappa_{m}\right) L / 2+s_{1} \pi(n+1 / 2)+s_{2} \pi(m+1 / 2)\right]}{\left(\kappa_{n}+\kappa_{m}\right) L / 2+s_{1} \pi(n+1 / 2)+s_{2} \pi(m+1 / 2)} .
$$

With the abbreviation $f(x)=\sin (x) / x(3.35)$ can therefore be rewritten as

$$
\begin{array}{r}
\frac{1}{\xi}=\frac{1}{l_{e l}} \sum_{s_{1} \ldots s_{4}} \sum_{n m} \frac{1}{\eta_{n} \eta_{m}} f\left(\pi\left[\eta_{n}+\eta_{m}+s_{1}(n+1 / 2)+s_{2}(m+1 / 2)\right]\right) \\
f\left(\pi\left[\eta_{n}+\eta_{m}+s_{3}(n+1 / 2)+s_{4}(m+1 / 2)\right]\right)
\end{array}
$$


With the help of the approximation scheme

$$
\begin{aligned}
\sum_{n=0}^{N-1} \frac{1}{\eta_{n}} h(n+1 / 2) & \approx \int_{0}^{N} \frac{d x}{\sqrt{N^{2}-x^{2}}} h(x) \\
& =\int_{0}^{1} \frac{d s}{\sqrt{1-s^{2}}} h(N s) \\
& =\int_{0}^{\pi / 2} d \varphi h(N \sin \varphi)
\end{aligned}
$$

for an arbitrary function $h(n+1 / 2)$ we find

$$
\begin{array}{r}
\frac{1}{\xi}=\frac{1}{l_{e l}} \sum_{s_{1} \ldots s_{4}} \int_{0}^{\pi / 2} d \varphi_{1} d \varphi_{2} f\left(\pi N\left[\cos \varphi_{1}+\cos \varphi_{2}+s_{1} \sin \varphi_{1}+s_{2} \sin \varphi_{2}\right]\right) \\
f\left(\pi N\left[\cos \varphi_{1}+\cos \varphi_{2}+s_{3} \sin \varphi_{1}+s_{4} \sin \varphi_{2}\right]\right)
\end{array}
$$

The main contributions to the integral arise from the regions where $[..] \approx 1 / N$. Also, the two factors in the integrand should be in phase, i.e. $s_{1}=s_{3}$ and $s_{2}=s_{4}$ (diagonal approximation). Therefore we are left with the expression (using $\sin \varphi+\cos \varphi=\sin (\varphi+$ $\pi / 4) / \sqrt{2})$

$$
\frac{1}{\xi}=\frac{1}{l_{e l}} \int_{-\pi / 2}^{\pi / 2} d \varphi_{1} d \varphi_{2} f^{2}\left(\frac{\pi N}{\sqrt{2}}\left[\sin \left(\varphi_{1}+\pi / 4\right)+\sin \left(\varphi_{2}+\pi / 4\right)\right]\right),
$$

for which the integration range can be restricted to a narrow strip around the line $\varphi_{1}=$ $-\pi / 2-\varphi_{2}$. A first order Taylor expansion in the deviation $\Delta \varphi$ from this line yields

$$
\frac{1}{\xi} \approx \frac{1}{l_{e l}} \int_{-\pi / 2}^{0} d \varphi \int_{-i n f t y}^{\infty} d(\Delta \varphi) f^{2}\left(\frac{\pi N}{\sqrt{2}} \cos (\varphi+\pi / 4) \Delta \varphi\right)
$$

With the help of the integral

$$
\int_{-\infty}^{\infty} d x \frac{\sin ^{2}(a x)}{(a x)^{2}}=\frac{\pi}{|a|}
$$

we get

$$
\begin{aligned}
\frac{1}{\xi} & =\frac{\pi}{l_{e l}} \int_{-\pi / 2}^{0} d \varphi \frac{\sqrt{2}}{\pi N} \frac{1}{|\cos (\varphi+\pi / 4)|} \\
& =\frac{\sqrt{2}}{N l_{e l}} \int_{-\pi / 4}^{\pi / 4} \frac{d \varphi}{\cos \varphi} \\
& =\frac{\sqrt{2}}{N l_{e l}} \ln \frac{\tan (3 \pi / 8)}{\tan (\pi / 8)}=\frac{2 \sqrt{2}}{N l_{e l}} \ln (1+\sqrt{2})
\end{aligned}
$$

which is exactly 3.36$)$. 


\section{REFERENCES}

[1] D. C. Thouless, Phys. Rev. Lett. 39, 1167 (1977).

[2] E. Abrahams, P. W. Anderson, D. C. Licciardello, and T. V. Ramakrishnan, Phys. Rev. Lett. 42, 673 (1979).

[3] P. W. Anderson, D. J. Thouless, E. Abrahams, and D. S. Fisher, Phys. Rev. B 15, 3519 (1980).

[4] F. J. Wegner, Z. Physik B 35, 207 (1979).

[5] K.B. Efetov, Adv. in Phys. 32, 53 (1983).

[6] F. J. Wegner, Nucl. Phys. B 316, 663 (1989).

[7] S. Hikami, Prog. Theor. Phys. 107, 213 (1992).

[8] K. B. Efetov and A. I. Larkin, Zh. Eksp. Theor. Fiz. 85, 764 (1983); Sov. Phys. JETP 58, 444 (1983).

[9] M. R. Zirnbauer, Phys. Rev. Lett. 69, 1584 (1992).

[10] A. D. Mirlin, A. Müller-Groeling, and M. R. Zirnbauer, Ann. Phys. (N.Y.) 236, 325 (1994).

[11] O. N. Dorokhov, Pis'ma Zh. Eksp. Teor. Fiz. 36, 259 (1982) [JETP Lett. 36, 318 (1982)]; P. A. Mello, P. Pereyra, and. N. Kumar, Ann. Phys. (N. Y.) 181, 290 (1988).

[12] C. W. J. Beenakker and B. Rejaei, Phys. Rev. Lett. 71, 3689 (1993); C. W. J. Beenakker and B. Rejaei, Phys. Rev. B 49, 7499 (1994).

[13] K. Frahm, Phys. Rev. Lett. 74, 4706 (1995).

[14] B. Rejaei, Phys. Rev. B 53, R13235 (1996).

[15] P. W. Brouwer and K. Frahm, Phys. Rev. B 53, 1490 (1996).

[16] D. L. Shepelyansky, Phys. Rev. Lett. 73, 2607 (1994).

[17] Y. Imry, Europhys. Lett. 30, 405 (1995).

[18] K. Frahm, A. Müller-Groeling, J.- L. Pichard, and D. Weinmann, Europhys. Lett. 31, 169 (1995).

[19] D. Weinmann, A. Müller-Groeling, J.-L. Pichard, and K. Frahm, Phys. Rev. Lett. 75, $1598(1995)$.

[20] F. von Oppen, T. Wettig, and J. Müller, Phys. Rev. Lett. 76, 491 (1996).

[21] F. Borgonovi and D. L. Shepelyansky, Nonlinearity 8, 877 (1995).

[22] F. Borgonovi and D. L. Shepelyansky, J. Phys. I France 6, 287 (1996).

[23] K. Frahm, A. Müller-Groeling, and J.- L. Pichard, Phys. Rev. Lett. 76, 1509 (1996).

[24] O. N. Dorokhov, Zh. Eksp. Teor. Fiz. 98, 646 (1990) [Sov. Phys. JETP 71, 360 (1990)].

[25] Y. Imry and J.- L. Pichard, Saclay preprint.

[26] P. Jacquod and D. L. Shepelyansky, Phys. Rev. Lett. 75, 3501 (1995).

[27] Y. V. Fyodorov and A. D. Mirlin, Phys. Rev. B 52, R11580 (1995).

[28] K. Frahm and A. Müller-Groeling, Europhys. Lett. 32, 385 (1995).

[29] J. J. M. Verbaarschot, H. A. Weidenmüller, and M. R. Zirnbauer, Phys. Rep. 129, 367 (1985).

[30] S. Iida, H.A. Weidenmüller, and J. A. Zuk, Ann. Phys. (N.Y.) 200, 219 (1990).

[31] Y. V. Fyodorov and A. D. Mirlin, Phys. Rev. Lett. 67, 2405 (1991); ibid. 69, 1093 (1992); ibid. 71, 412 (1993).

[32] M. L. Mehta, Random Matrices, 2nd ed. (Academic, New York, 1991).

[33] B. L. Al'tshuler and B. I. Shklovskii, Zh. Eksp. Teor. Fiz. 91, 220 (1986) [Sov. Phys. JETP 64, 127 (1986)]. 
[34] F. von Oppen and T. Wettig, Europhys. Lett. 32, 741 (1995).

[35] E. Akkermans and J.-L. Pichard, preprint (1996).

[36] A. Müller-Groeling, H.A. Weidenmüller and C.H. Lewenkopf, Europhys. Lett. 22, 193 (1993); A. Müller-Groeling and H.A. Weidenmüller, Phys. Rev. B 49, 4752 (1994). 